\title{
Efficient Long-range, Directional Energy Transfer through DNA-Templated Dye Aggregates
}

Xu Zhou,, 1,3, \$ Sarthak Mandal,,${ }^{2,5, ~}$ Shuoxing Jiang, ${ }^{1}$ Su Lin, ${ }^{2,3}$ Jianzhong Yang, ${ }^{4}$ Yan Liu, ${ }^{1,3}$ David G. Whitten, ${ }^{4}$ Neal W. Woodbury ${ }^{2,3 *}$ and Hao Yan ${ }^{1,3 *}$

${ }^{1}$ Center for Molecular Design and Biomimetics at the Biodesign Institute, Arizona State University,

Tempe, Arizona, USA

${ }^{2}$ Center for Innovations in Medicine at the Biodesign Institute, Arizona State University, Tempe, Arizona, USA

${ }^{3}$ School of Molecular Sciences, Arizona State University, Tempe, Arizona, USA

${ }^{4}$ Department of Chemical and Biological Engineering, University of New Mexico, Albuquerque, New Mexico, USA

${ }^{5}$ Department of Chemistry, National Institute of Technology Tiruchirappalli, Tamil Nadu, India *Corresponding Authors (nwoodbury@asu.edu, hao.yan@asu.edu)

${ }^{\$}$ Both these authors contributed equally to this work 


\section{Table of Contents}

Section 1. Experimental materials and methods

Section 2. Additional spectra

Section 3. DNA sequences and characterization of DNA-dye conjugation

Section 4. Calculation of energy transfer efficiency

Section 5. Estimation of overall through bridge donor-to-acceptor energy transfer efficiency

Section 6. A simple kinetics model of D-B-A energy transfer system 


\section{Section 1. Experimental materials and methods}

\subsection{Materials}

All DNA strands were purchased from Integrated DNA Technologies Inc. and purified by denaturing polyacrylamide gel electrophoresis. The benzothiazole cyanine dye was obtained from Dr. David Whitten, University of New Mexico. ${ }^{1}$ Alexa fluor 350 NHS ester (AF350-NHS), Alexa fluor 555 NHS ester (AF555NHS) and Alexa fluor 555 C2 maleimide (AF555-maleimide) were purchased from Thermal Fisher Scientific. All chemicals were used as received without further purification.

\subsection{K21 dye stock solution}

The K21 stock solution was prepared by dissolving K21 dye powder into DMSO to reach a $1.67 \mathrm{mM}$ concentration of K21, which was stored at room temperature in the dark. The final concentration of K21 stock solution was checked by absorption measurement using molar extinction coefficient of $92,366.7 \mathrm{M}^{-}$ ${ }^{1} \mathrm{~cm}^{-1}$ at $433 \mathrm{~nm} .{ }^{1}$

\subsection{Fluorophore-DNA conjugation}

The fluorophore-DNA conjugates were generally prepared by using amine-NHS ester reaction. In a typical amine-NHS ester conjugation reaction, the amine modified DNA $(0.25 \mathrm{mM}, 50 \mu \mathrm{L})$ was mixed with fluorophore NHS ester (16 mM in DMSO, $15.6 \mu \mathrm{L})$ and sodium bicarbonate buffer $(1 \mathrm{M}, \mathrm{pH} 8.4,10 \mu \mathrm{L})$. The mixture was placed on a shaker for 2 hours at room temperature and then purified by an Agilent Technologies 1200 series reverse phase HPLC system using a ZORBAX SB-C18 column (solvent A: TEAA (trimethylamine acetate) buffer, $100 \mathrm{mM}, \mathrm{pH}$ 7.0; solvent B: acetonitrile, flow rate: $1 \mathrm{~mL} / \mathrm{min}$ ). The collected fraction was lyophilized and re-dissolved in distilled water. The acceptor labeled DNA strands for constructs with lengths of $30 \mathrm{bp}, 60 \mathrm{bp}$ and $100 \mathrm{bp}$ were prepared by thiol-maleimide conjugation. In a typical thiol-maleimide conjugation reaction, the thiol group modified DNA $(0.25 \mathrm{mM}, 50 \mu \mathrm{L})$ was mixed with TCEP (tris(2-carboxyethyl)phosphine) $(100 \mathrm{mM}, \mathrm{pH} 7.0,2.5 \mu \mathrm{L})$ and sodium phosphate buffer (400 $\mathrm{mM}, \mathrm{pH} 7.0,10 \mu \mathrm{L})$. The mixture was placed on a shaker for 2 hours at room temperature. And Alexa fluor $555 \mathrm{C} 2$ maleimide $(16 \mathrm{mM}$ in DMSO, 15. $6 \mu \mathrm{L})$ was added and the mixture was placed on a shaker for 2 hours at room temperature. The dye-DNA conjugates were purified, lyophilized and re-dissolved as 
described above. The dual-labeled AF350-DNAs-AF555 for $100 \mathrm{bp}$ construct was prepared by using thiolgroup and amino-group dual-modified DNA strand. First, the thiol-DNA-amine was conjugated with AF555-maleimide following the thiol-maleimide conjugation protocol and then the conjugate was purified by using 3K Amicon ultra centrifugal filters. The purified AF555-DNA was reacted with AF350-NHS following the amino-NHS ester conjugation protocol and then dual-dye labeled DNA strand was purified by reverse phase-HPLC. All fluorophore-DNA conjugates were characterized by mass spectrometry using AB SCIEX 4800 system (given in the Section 3).

\subsection{Preparation of double stranded DNA (dsDNA) template}

Equal molar amount of complementary single stranded DNAs (ssDNAs) were mixed in the Tris-acetate buffer (40 mM Tris-acetate, $0.5 \mathrm{mM}$ magnesium acetate, $\mathrm{pH} 8.4$ ) to give a final DNA concentration of 4 $\mu \mathrm{M}$. The mixture was heated to $95^{\circ} \mathrm{C}$ for $3 \mathrm{~min}$ and then cooled to room temperature to form the designed dsDNA template.

\subsection{Preparation of DNA-templated dye aggregates}

In a typical experiment, the DNA-templated K21 dye aggregates were prepared by simply mixing K21 stock solution and dsDNAs solution in the molar ratio of 1:4 (dye to base pairs) at a final k21 dye concentration of $30 \mu \mathrm{M}$.

\subsection{Spectroscopic characterization}

\section{6a. Steady-state spectroscopy}

Absorption spectra were measured on a Jasco V-670 spectrophotometer using a quartz cuvette of $3 \mathrm{~mm}$ path length. Steady state fluorescence emission and excitation spectra were measured on a Nanolog fluorometer (Horiba Jobin Yvon) using a quartz cuvette of $3 \mathrm{~mm}$ path length and corrected for the wavelength dependence of the detection system response. The inner filter effect on the fluorescence intensity was corrected by using the following equation:

$$
I_{\text {corr }}=I \times 10^{(0.5 \times A)}
$$


Where $I$ is the observed fluorescence intensity at certain wavelength, $A$ is the absorbance of the sample at the same wavelength, and $I_{\text {corr }}$ is the corrected fluorescence intensity.

\section{6b. Time-correlated single photon counting}

Fluorescence decay kinetics was measured using time-correlated single-photon counting (TCSPC). The excitation source was a titanium sapphire (Ti:S) laser (Spectra-Physics, Millennia pumped Tsunami) with a $130 \mathrm{fs}$ pulse duration operated at $82 \mathrm{MHz}$. The laser output was sent through a pulse selector and a frequency doubler (Spectra Physics, Model 3980) to obtain excitation pulses at $350 \mathrm{~nm}$ with $4 \mathrm{MHz}$ repetition rate. Fluorescence emission was collected at a $90^{\circ}$ geometry setting and detected using a doublegrating monochromator (Jobin-Yvon, Gemini-180) coupled to a microchannel plate photomultiplier tube (Hamamatsu R3809U-50). The polarization of the emission was set at $54.7^{\circ}$ relative to that of the excitation. Data acquisition used a single photon counting card (Becker-Hickl, SPC-830). The typical instrument response function had a full width at the half maximum of $40 \mathrm{ps}$, measured from the scattered light from a sample at $350 \mathrm{~nm}$. The decay data was fitted either globally with a sum of exponential decay model, or at a single wavelength using a home-written program ASUFIT.

\section{6c. Femtosecond transient absorption spectroscopy}

Femtosecond transient absorption spectra were recorded using a commercial spectrometer (HELIOS, Ultrafast Systems, Sarasota, FL). The excitation source is a kilohertz Titanium:Sapphire laser described previously. Briefly, laser pulses of $1 \mathrm{~mJ}$, at a repetition rate of 1 kilohertz (100 fs pulse duration at $800 \mathrm{~nm})$, were generated from a regenerative amplifier system (Tsunami and Spitfire, Spectra-Physics). Part of the pulse energy $(\sim 600 \mu \mathrm{J})$ was sent to an optical parametric amplifier to generate the excitation pulses at 350 $\mathrm{nm}$, and the rest part was sent to the HELIOS system to generate a broad-band probe spectrum. All of the experiments were performed at room temperature. The recorded transient absorption changes as a function of wavelength and time were analyzed with a locally-written program, ASUFIT, using equation $F(\lambda)=\sum_{i} A_{i}(\lambda) e^{-t / \tau_{i}}$. The instrument response function was fit to a Gaussian curve with a full-width at 
half maximum of $100-120 \mathrm{fs}$, and the group velocity dispersion of the white light probe pulse was fitted to a third-order polynomial.

\section{Section 2. Additional spectra}
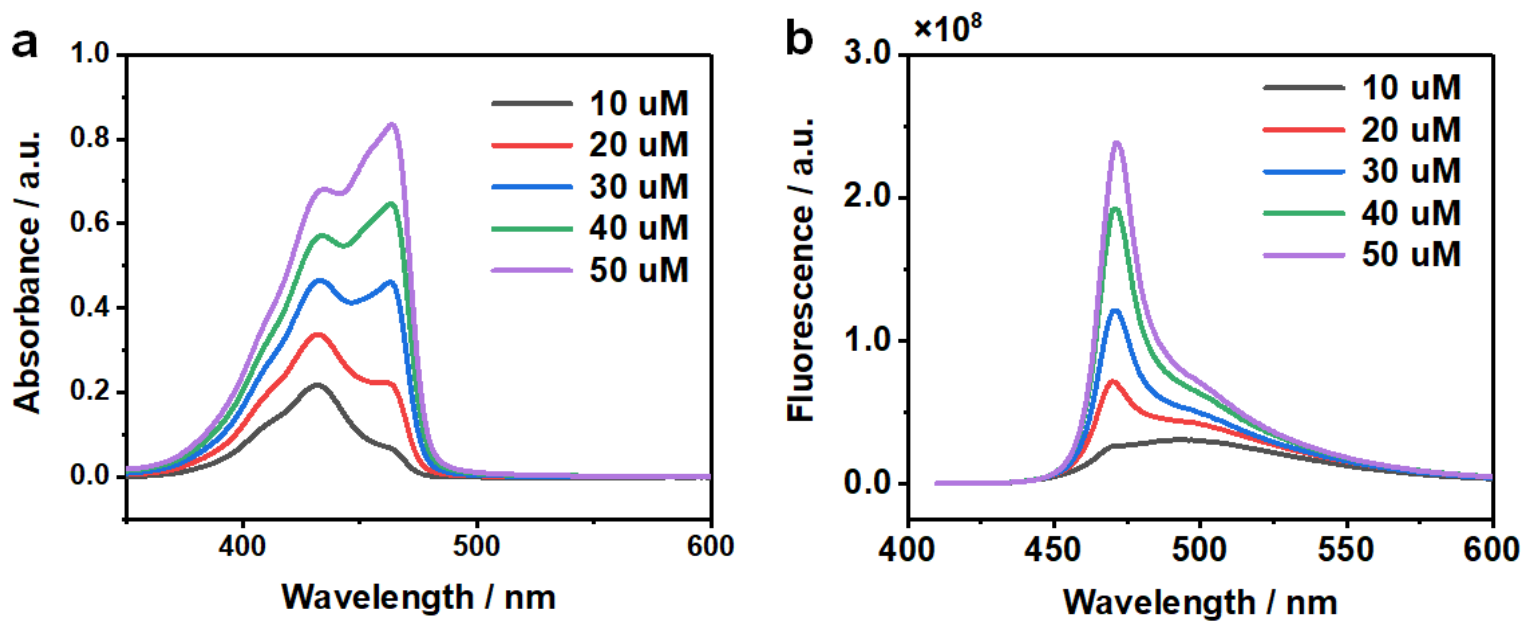

Figure S1. Spectral properties of K21 dye aggregates on $4 \mu \mathrm{M}$ poly $(\mathrm{A})_{24} /$ poly $(\mathrm{T})_{24}$ dsDNA $(30 \mathrm{bp})$ in the presence of different dye concentration conditions. a. Absorption spectra and b. Steady-state fluorescence emission spectra of the dye aggregates as the dye concentration increases from 10 to $50 \mu \mathrm{M}$ while DNA concentration kept constant. All of the fluorescence spectra were measured with excitation at $350 \mathrm{~nm}$. While it is possible to fit this data to a cooperative binding model and derive apparent dissociation constants in the tens of micromolar range, such an analysis is only a rough estimate. We cannot increase the concentrations to high enough levels to saturate the system without introducing measurement artifacts (free dye absorbance/self absorption of fluorescence) and we are also in a range where simple binding models fail (the concentration of binding sites is similar to the dye concentration).
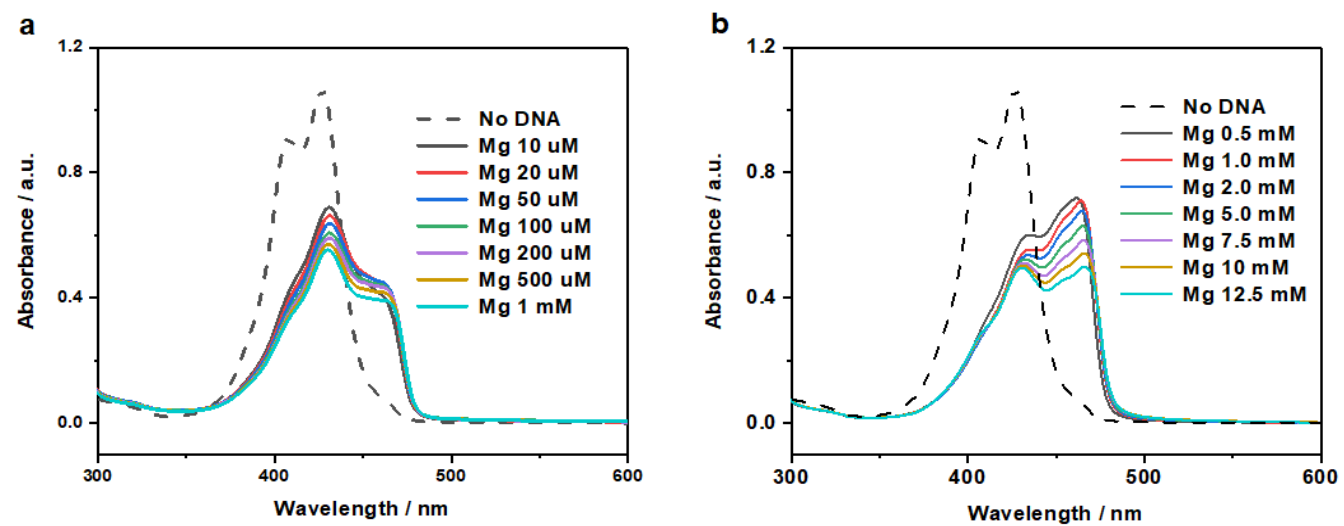

Figure S2. Absorption spectra of K21 aggregates templated on $4 \mu \mathrm{M}$ poly $(\mathrm{A})_{24} / \operatorname{poly}(\mathrm{T})_{24}$ dsDNA under different ionic conditions of the solution. a. $\mathrm{Mg}^{2+}$ concentration increased from $10 \mu \mathrm{M}$ to $1 \mathrm{mM}$ at a constant $\mathrm{K} 21$ concentration of $30 \mu \mathrm{M}$. b. $\mathrm{Mg}^{2+}$ concentration increased from $0.5 \mathrm{mM}$ to $12.5 \mathrm{mM}$ at a constant $\mathrm{K} 21$ concentration of $50 \mu \mathrm{M}$. 

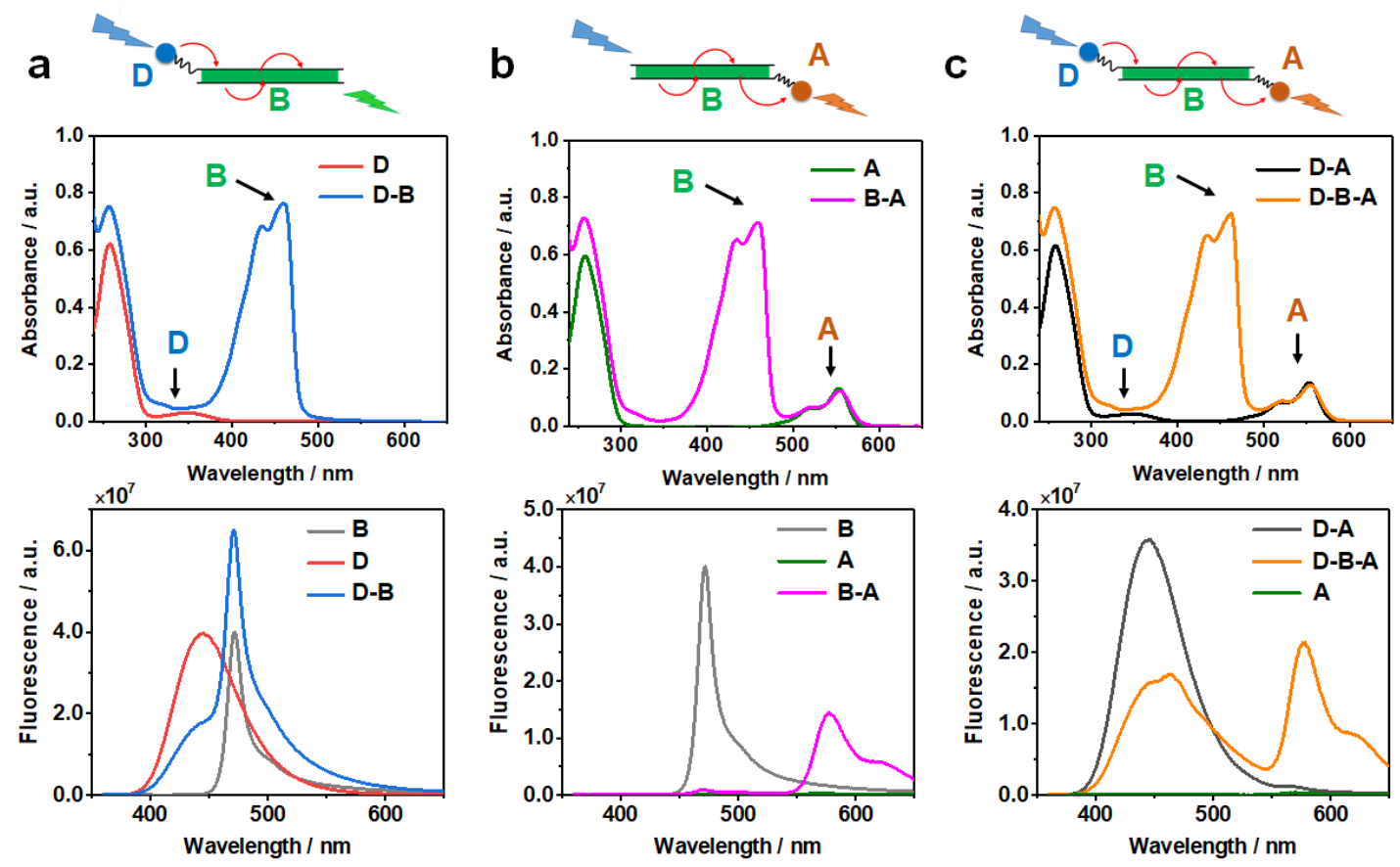

Figure S3. Scheme and characterization of energy transfer process in the one-donor D-B-A system. a. Absorption spectra (top panel) of construct D and D-B. The two-component energy transfer from the donor to the bridge (bottom panel) is characterized by steady-state fluorescence spectroscopy. $\mathbf{b}$. Absorption spectra (top panel) of construct A and B-A. The two-component energy transfer from the aggregates bridge to the acceptor (bottom panel) is characterized by the steady-state fluorescence spectroscopy. c. Absorption spectra (top panel) of construct D-A and D-B-A. The three-component energy transfer from donor to acceptor through aggregates (bottom panel) is characterized by steady-state fluorescence spectroscopy. All samples are excited at $350 \mathrm{~nm}$ and all emission spectra are corrected by the inner filter effect. 

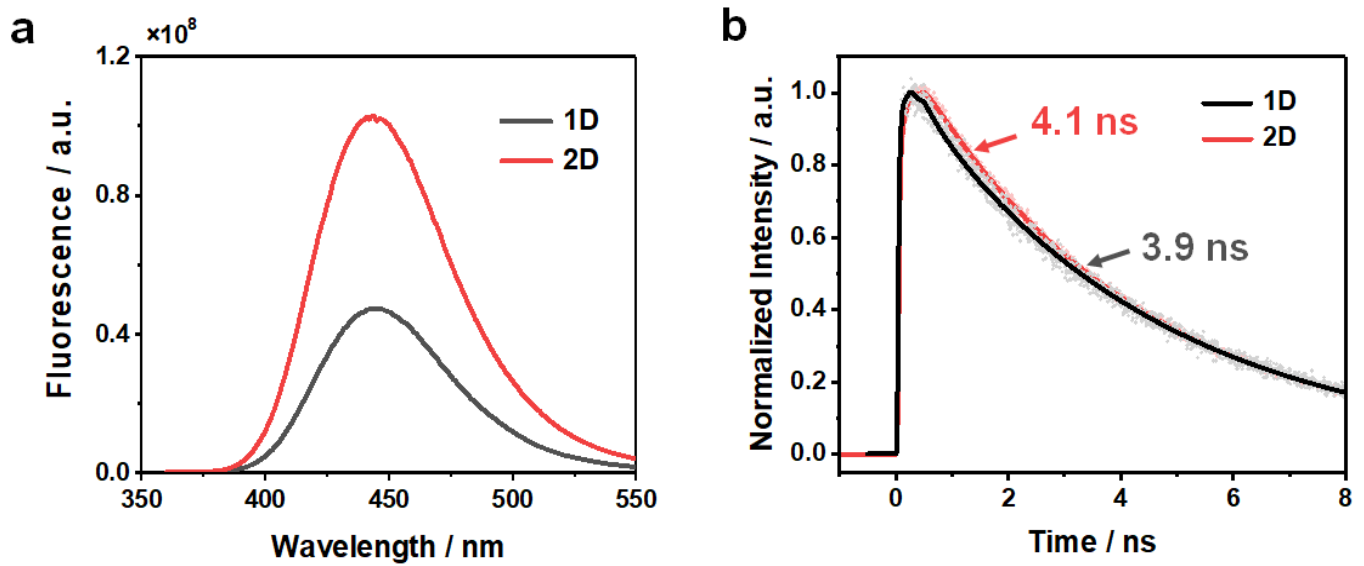

Figure S4. Comparison of fluorescence spectral properties of donor in one-donor (1D) system and twodonor (2D) system templated on poly(A) $)_{24} /$ poly(T) ${ }_{24}$ of the same DNA concentration. a. Steady-state fluorescence spectra of 1D (black line) and 2D (red line). The fluorescence intensity of 2D at $450 \mathrm{~nm}$ is 2.16 times larger than that of 1D. b. Normalized fluorescence decay kinetic traces recorded at $450 \mathrm{~nm}$ of 1D (black line) and 2D (red line) shown the fluorescence decay lifetime of 3.9 ns and $4.1 \mathrm{~ns}$ respectively. All experiments were carried out with the excitation at $350 \mathrm{~nm}$. The minor lifetime change suggests that self-quenching of two donors is negligible in the $2 \mathrm{D}$ system. 
a

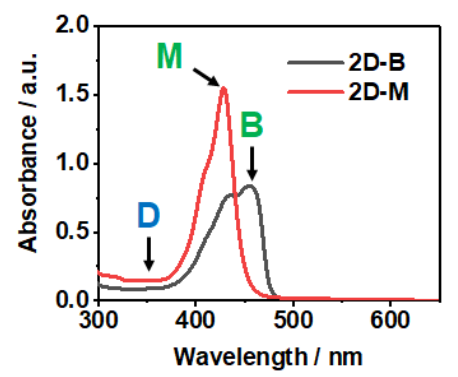

b
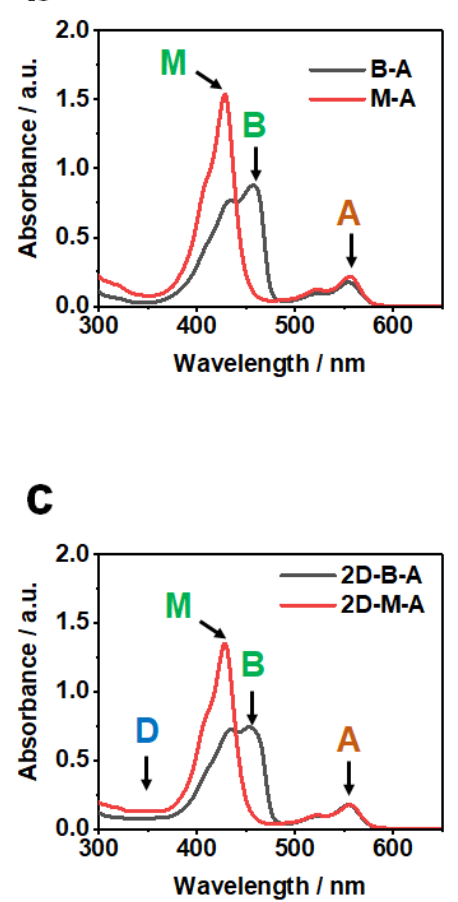
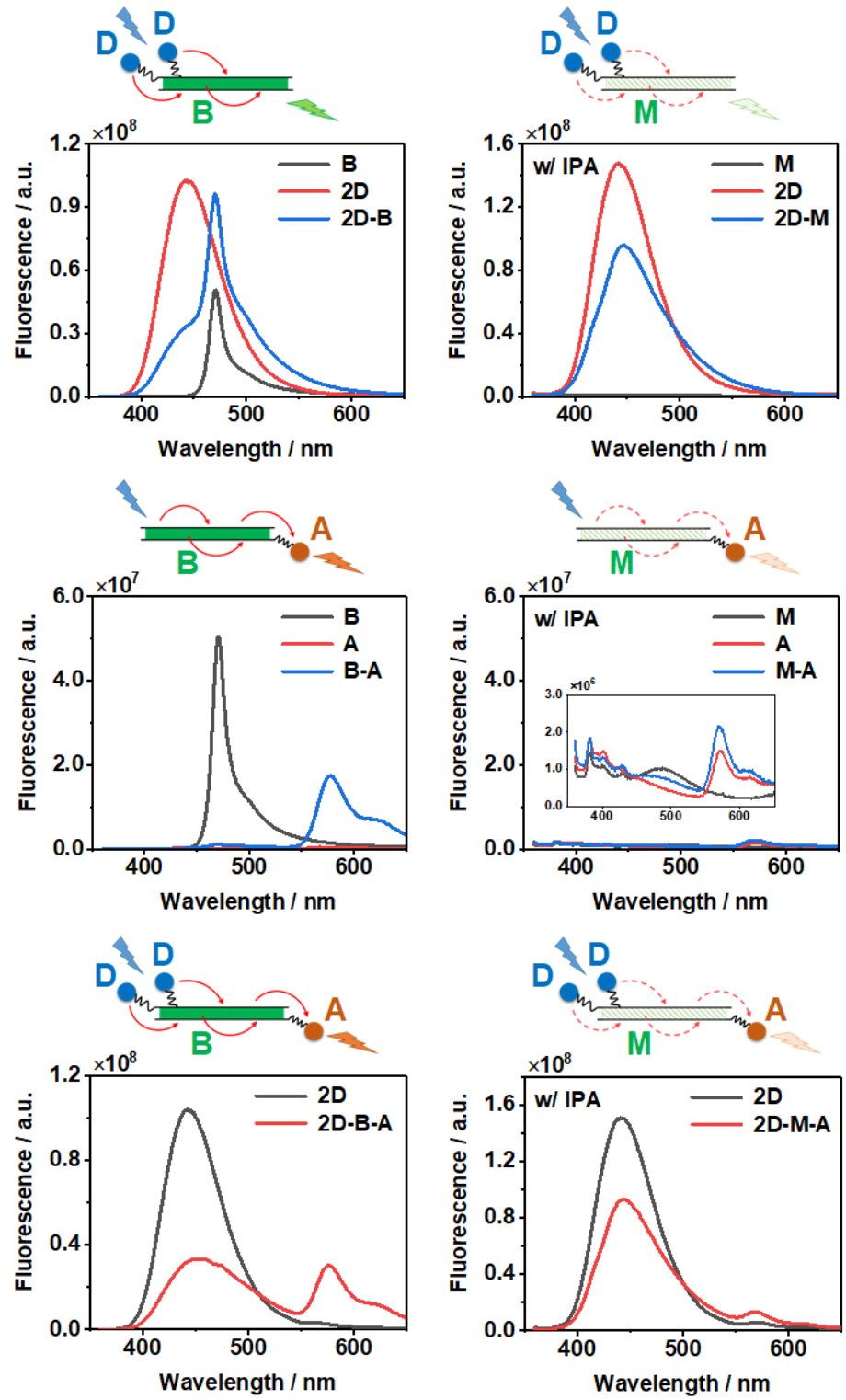

Figure S5. Scheme and characterization of absorbance (left panel) and energy transfer processes of twodonor system (middle panel) in comparison with the negative control (right panel) without a proper formation of the K21 aggregates. In the negative control group, we kept the same construct design, same concentrations of $\mathrm{K} 21$ dyes and DNA, but used a different buffer solution containing 30\% isopropanol (IPA), which does not interrupt the DNA duplex formation but preserves K21 in its monomeric (M) form in solution thus prevents formation of the dye aggregates on the DNA template. a. Absorption spectra (left panel) of the constructs 2D-B and 2D-M, and steady-state fluorescence spectroscopy characterization of the two-component energy transfer from donor to K21 aggregates bridge on the DNA template (B, middle panel) or K21 monomer in solution (M, right panel). b. Absorption spectra (left panel) of the constructs B$\mathrm{A}$ and $\mathrm{M}-\mathrm{A}$, and steady-state fluorescence spectroscopy characterization of the two-component energy transfer from aggregates ( $\mathrm{B}$, middle panel) or $\mathrm{K} 21$ monomer (M, right panel) to acceptor. c. Absorption spectra (left panel) of the constructs 2D-B-A and 2D-M-A, and steady-state fluorescence spectroscopy characterization of the three-component energy transfer from donor to acceptor mediated by aggregates (B, middle panel) or K21 monomer (M, right panel). All samples are excited at $350 \mathrm{~nm}$. 
a
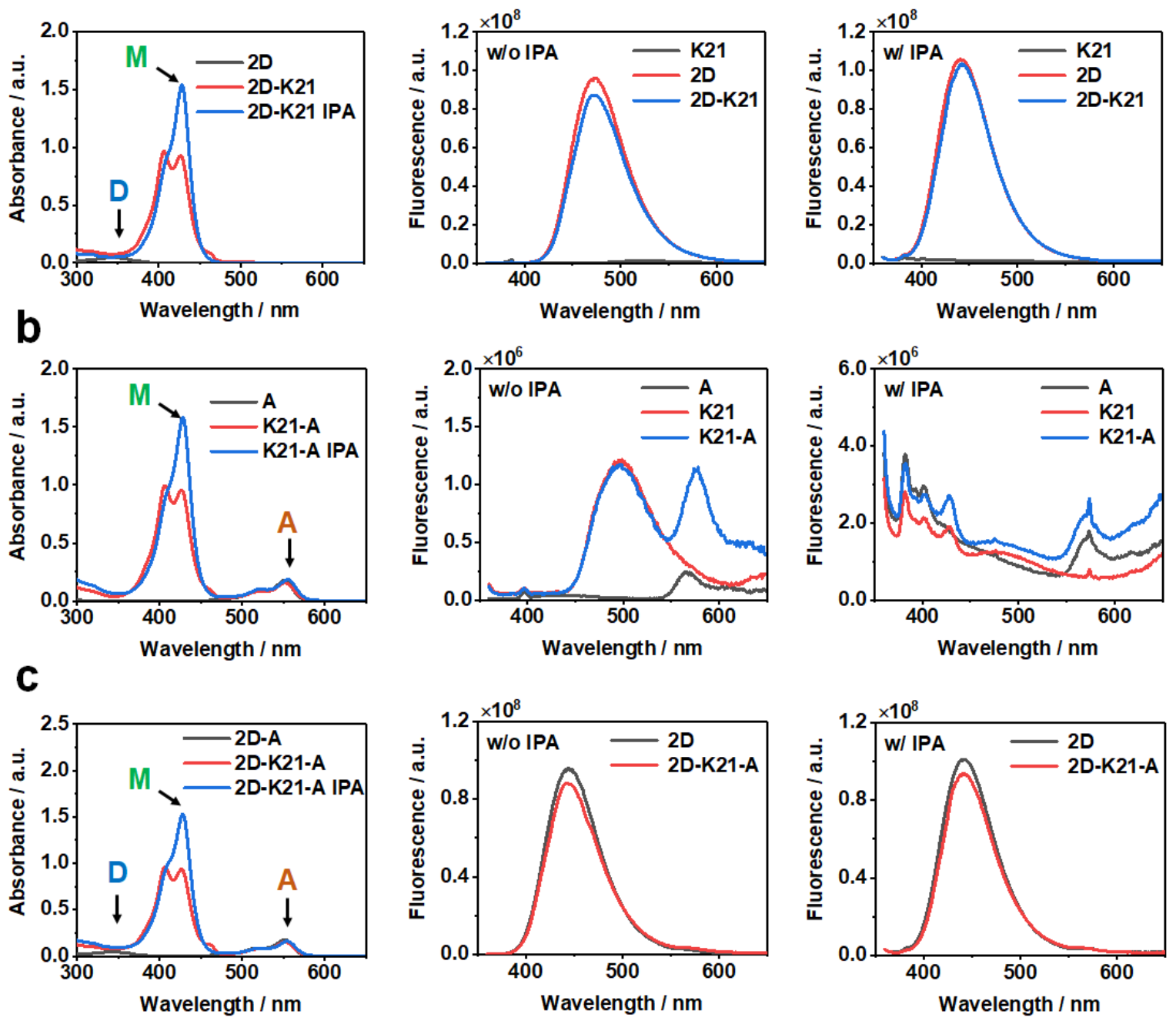

Figure S6. Characterization of energy transfer processes of no-DNA negative control groups (various simple mixtures of 2D, K21 and A), where we designed the systems without the DNA templates and kept the concentrations of donor, acceptor and K21 dye the same as those in the 2D-B-A DNA-templated construct. One group was designed to use the normal buffer (middle panel), and the other was designed to use the buffer containing 30\% isopropanol (IPA, right panel). a. Absorption (left panel) and fluorescence emission spectra (middle and right panel) of various 2D-K21 mixtures with and without IPA. b. Absorption spectra (left panel) and fluorescence emission spectra (middle and right panel) of various K21-A mixture with and without IPA. c. Absorption spectra (left panel) and fluorescence emission spectra (middle and right panel) of various mixture of 2D-K21-A with and without IPA. 

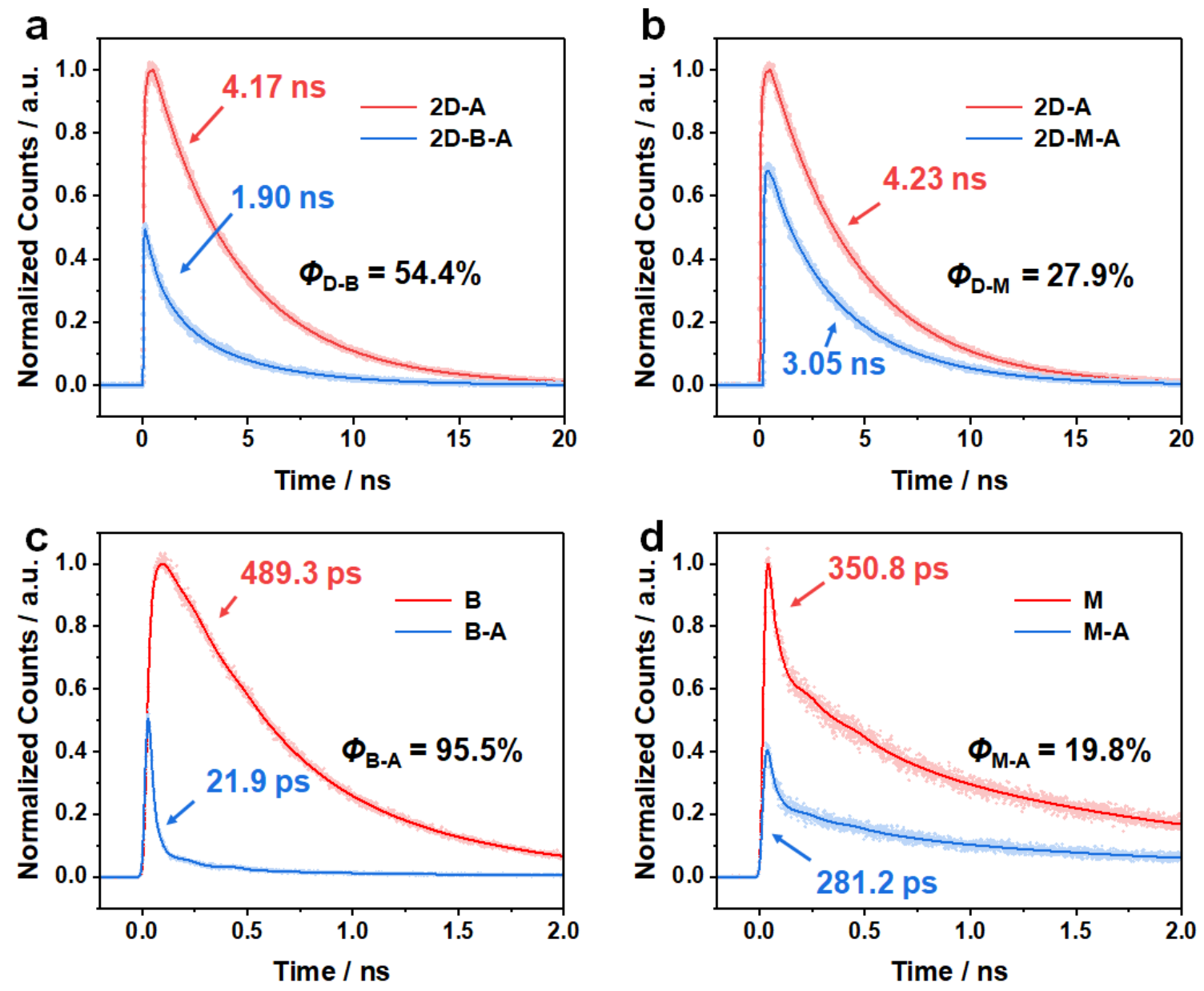

Figure S7. Comparison of fluorescence decay kinetics in 2D-B-A and 2D-M-A systems. a. The fluorescence decay kinetics of 2D-A (red line) and 2D-B-A (blue line) are monitored at $450 \mathrm{~nm}$ (donor emission wavelength) and are normalized by dividing by the maximum amplitude of 2D-A. The average fluorescence lifetime $\tau$ of the donor in 2D-A or 2D-B-A construct is labeled in red and blue font, respectively. The energy transfer efficiencies are calculated using $1-\tau(2 \mathrm{D}-\mathrm{B}-\mathrm{A}) / \tau(2 \mathrm{D}-\mathrm{A})$. b. The Fluorescence decay kinetics of 2D-A (red line) and 2D-M-A (blue line) are monitored at $450 \mathrm{~nm}$ (donor emission wavelength) and are normalized by dividing by the maximum amplitude of 2D-A. The average fluorescence lifetime $\tau$ of donor in 2D-A or 2D-M-A construct is labeled as red and blue, respectively. The energy transfer efficiencies are calculated using 1- $\tau(2 \mathrm{D}-\mathrm{M}-\mathrm{A}) / \tau(2 \mathrm{D}-\mathrm{A})$. c. The Fluorescence decay kinetics of B (red line) and B-A (blue line) are monitored at $475 \mathrm{~nm}$ (bridge emission wavelength) and are normalized by dividing by the maximum amplitude of $\mathrm{B}$. The average fluorescence lifetime $\tau$ of bridge in $\mathrm{B}$ only or B-A construct is labeled as red and blue respectively. The energy transfer efficiencies are calculated using 1- $\tau(\mathrm{B}-\mathrm{A}) / \tau(\mathrm{B})$. d. The Fluorescence decay kinetics of $\mathrm{M}$ (red line) and M-A (blue line) are monitored at $475 \mathrm{~nm}$ (bridge emission wavelength) and are normalized by dividing by the maximum amplitude of M. The average fluorescence lifetime $\tau$ of bridge in M or M-A construct is labeled as red and blue respectively. The energy transfer efficiencies are calculated using 1- $\tau(\mathrm{M}-\mathrm{A}) / \tau(\mathrm{M})$. All samples were excited at $350 \mathrm{~nm}$. 


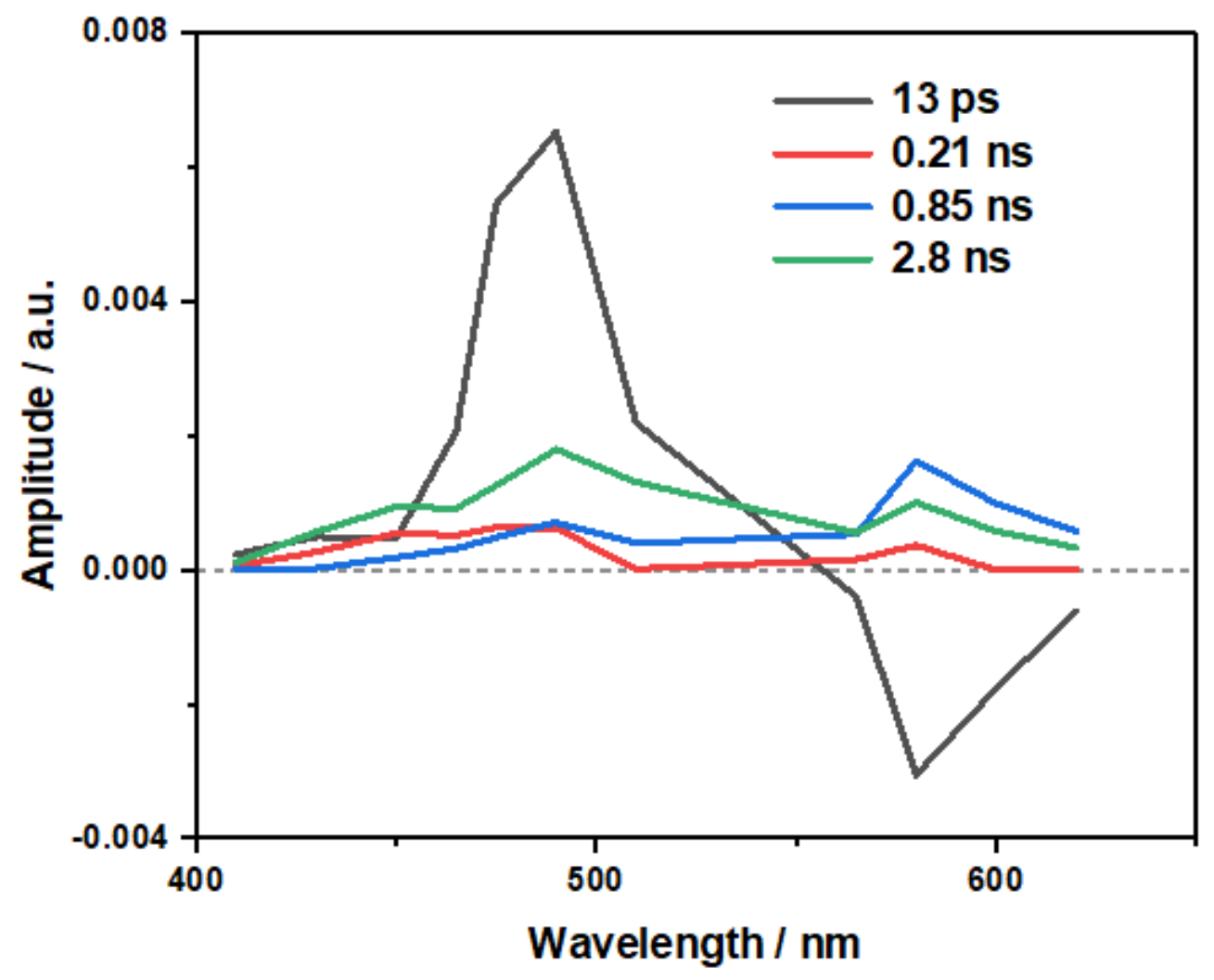

Figure S8. Decay-associate spectra obtained from global fitting of energy transfer in the 2D-B-A construct using sum of 4 exponential decay components over the wavelength from $410 \mathrm{~nm}$ to $620 \mathrm{~nm}$. The samples were excited at $350 \mathrm{~nm}$. 


\section{Detailed analysis by ultrafast transient absorbance spectroscopy}
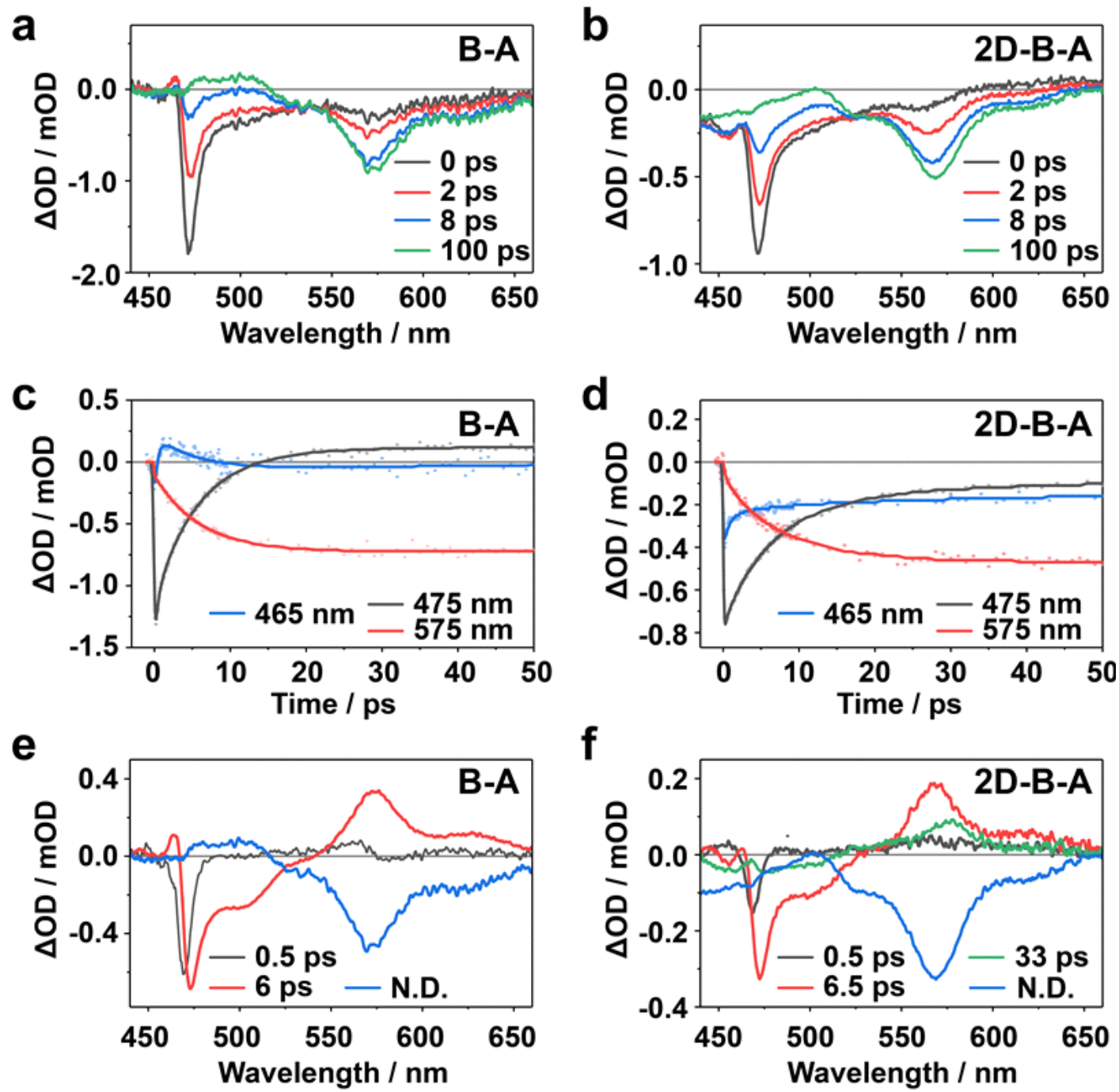

Figure S9. Transient absorption spectroscopic characterization of energy transfer processes in B-A and 2D-B-A constructs. Time-resolved absorption difference spectra at various delay times following excitation at $350 \mathrm{~nm}$ for a. B-A and b. 2D-B-A. Kinetic traces at $465 \mathrm{~nm}, 475 \mathrm{~nm}$, and $575 \mathrm{~nm}$ of c. B-A and d. 2D-B-A. Decay-associate spectra obtained from global fitting of transient absorption changes using e. 3 exponential decay components for B-A and f. 4 experientials for 2D-B-A. N.D. means non-decaying component.

The 0-ps spectrum of B-A shows a sharp bleaching band at $475 \mathrm{~nm}$ with a broad shoulder on the long wavelength side and a weak bleaching centered at $575 \mathrm{~nm}$, due to the formation of excited states of B and A, respectively. A clear transient decrease of the amplitude of the B-band accompanied with an increase of the A-band in the 0-8 ps time range suggests an efficient B-to-A energy transfer, which is largely completed within the first 8 ps (Figures S9a). The major difference of all time-resolved spectra in the 2D-B-A compared with those from the B-A is a broad bleaching background signal across the wavelength region of $440-500 \mathrm{~nm}$, superimposed in the absorption change signals of B and A excited states (Figures S9b). The absorption decrease is due to the stimulated emission of the donor, which span from 400 to $500 \mathrm{~nm}$. Similar to the spectral evolution observed in B-A, the 475-nm bleaching in 2D-B-A is largely recovered within the first $8 \mathrm{ps}$ and the bleaching at $575 \mathrm{~nm}$ is largely developed. However, the bleaching signal at the 
wavelengths below $500 \mathrm{~nm}$ persists beyond $100 \mathrm{ps}$, which represents the long-lived excited state of D. It is also noticed that a further absorption increase of the A-band between 8 and 100 ps in the 2D-B-A construct that is largely missing in the B-A construct, indicating a continued increase of the excited state population of $\mathrm{A}$ in the presence of the excited state of the donor, consistent with the long lifetime of the donor ( $\sim 4 \mathrm{~ns})$.

The kinetic traces of absorbance change at 475 and $575 \mathrm{~nm}$, correspond to the excited state population changes of B and A, respectively (Figure S9c), while the absorbance kinetics at $465 \mathrm{~nm}$ reflect the donor excited state kinetics in the 2D-B-A construct, which were also plotted as a reference for both constructs. The $465 \mathrm{~nm}$ kinetics in B-A shows a short-lived positive signal with a very similar kinetics of that of bleaching signal at $475 \mathrm{~nm}$, representing the excited absorption and ground state bleaching of B, respectively. The recovery of the signals at 465 and $475 \mathrm{~nm}$ are accompanied with a grow-in of the bleaching at $575 \mathrm{~nm}$, i.e. a population increase of the excited state of A. This B-to-A energy transfer process is completed within $20 \mathrm{ps}$. In contrast, the signal at $465 \mathrm{~nm}$ in 2D-B-A (Figure S9d) remains negative after a fast decay in the first few ps. The bleaching signal at $475 \mathrm{~nm}$ also exhibits a long-lived bleaching in addition to the recovery within the first $20 \mathrm{ps}$. There is a slow phase of absorption recovery at $475 \mathrm{~nm}$ and increase at $575 \mathrm{~nm}$ observed in 2D-B-A compared with that in B-A. Those results suggest that in addition to the rapid energy transfer from $\mathrm{B}$ to $\mathrm{A}$, there is an energy transfer process from $2 \mathrm{D}$ to $\mathrm{B}$ (then to $\mathrm{A}$ ) but at a much slower rate.

Global analysis of the spectral-temporal data reveals the energy transfer processes in the B-A and 2DB-A constructs. For B-A, 3 exponential components, with lifetimes of 0.5 ps, 6.0 ps, and a non-decaying (N.D., lifetime much longer than the 100-ps measuring window) components are resolved from the fitting to yield the dynamic associated spectrum (DAS) shown in Figure S9e. The 0.5 ps DAS exhibits a narrow bleaching at $470 \mathrm{~nm}$, likely due to a fast excited-state relaxation of B within the excitonic band. The negative signal at $475 \mathrm{~nm}$ and the positive signal at $565 \mathrm{~nm}$ in the 6-ps DAS are the typical characteristic spectral feature of energy transfer from B to A, and that the long-lived bleaching at $565 \mathrm{~nm}$ is due to the excited state of A, which lived for 2.4 ns as determined by the TCSPC measurement (Figure S9f). In contrast, the global fitting result of the 2D-B-A construct shows that besides the 3 DAS found in the B-A construct, an additional 33-ps DAS is necessary for an adequate fitting of the 2D-B-A data (Figure S9f, green curve). This DAS has the characteristic of energy transfer from 2D to A, likely via B. The much slower rate of energy transfer from $2 \mathrm{D}$ to $\mathrm{B}$ compared to that from $\mathrm{B}$ to A resulted in a low population of the excited state of $\mathrm{B}$ in the system as no accumulation of the excited $\mathrm{B}$ is expected. The non-decaying DAS shows a bleaching band at $575 \mathrm{~nm}$, representing the excited population of A, accompanied by a broad bleaching signal at wavelength below $500 \mathrm{~nm}$ due to the long-lived excited state of 2D. 
a
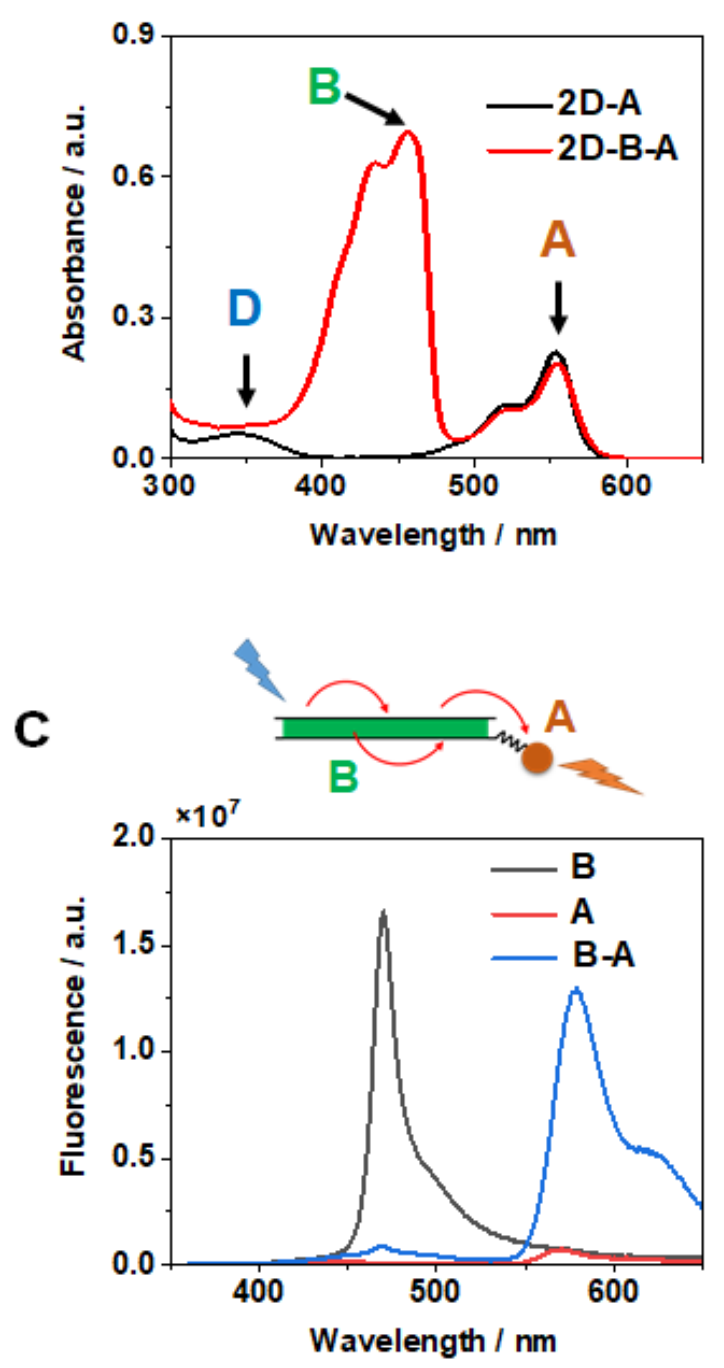
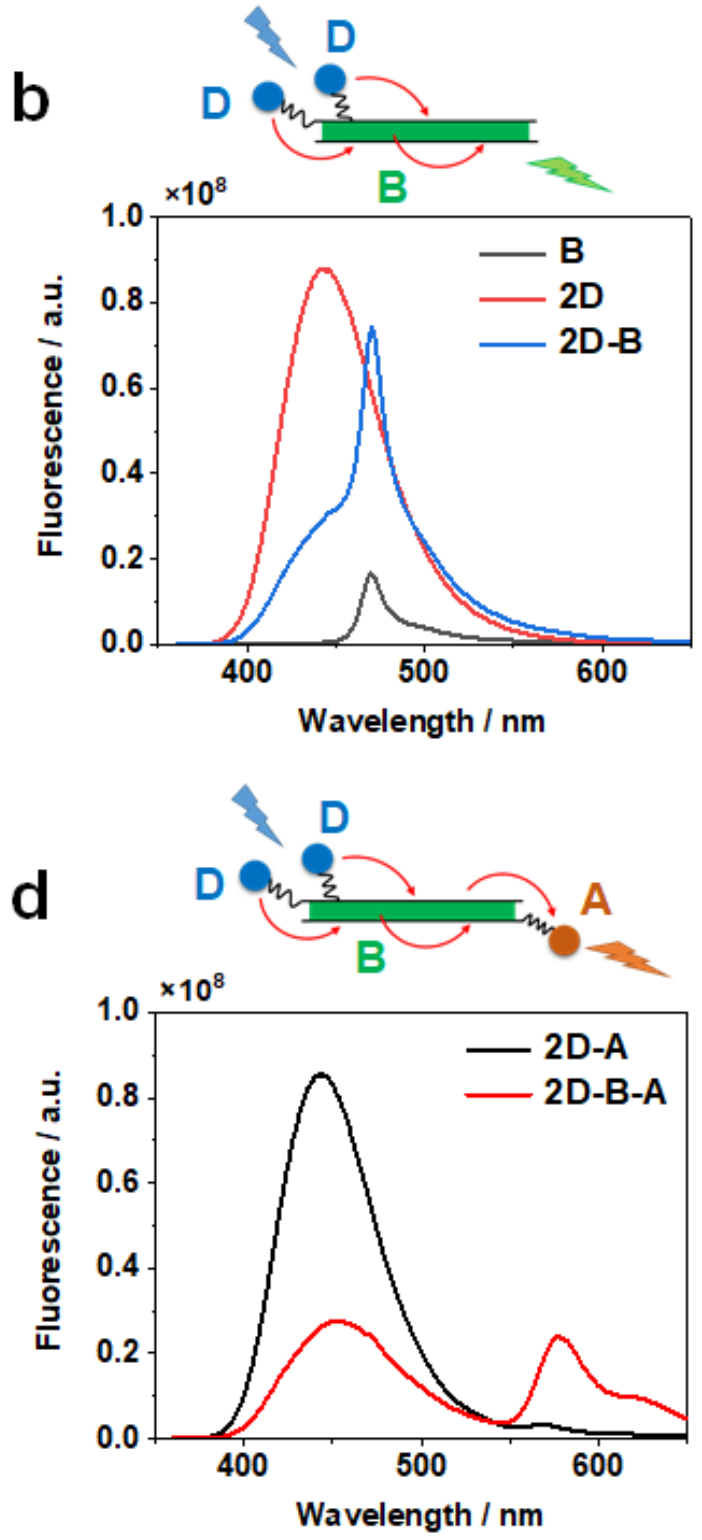

Figure S10. Energy transfer study of 2D-B-A system based on $30 \mathrm{bp}$ random sequence dsDNA template. a. Absorption spectra of constructs 2D-A (black line) and 2D-B-A (red line). b. Steady-state fluorescence spectra of construct B (black line), 2D (red line) and 2D-B (blue line). c. Steady-state fluorescence spectra of construct B (black line), A (red line) and B-A (blue line). d. Steady-state fluorescence spectra of construct 2D-A (black line) and 2D-B-A (red line). All fluorescence experiments were carried out with excitation at $350 \mathrm{~nm}$. 
a
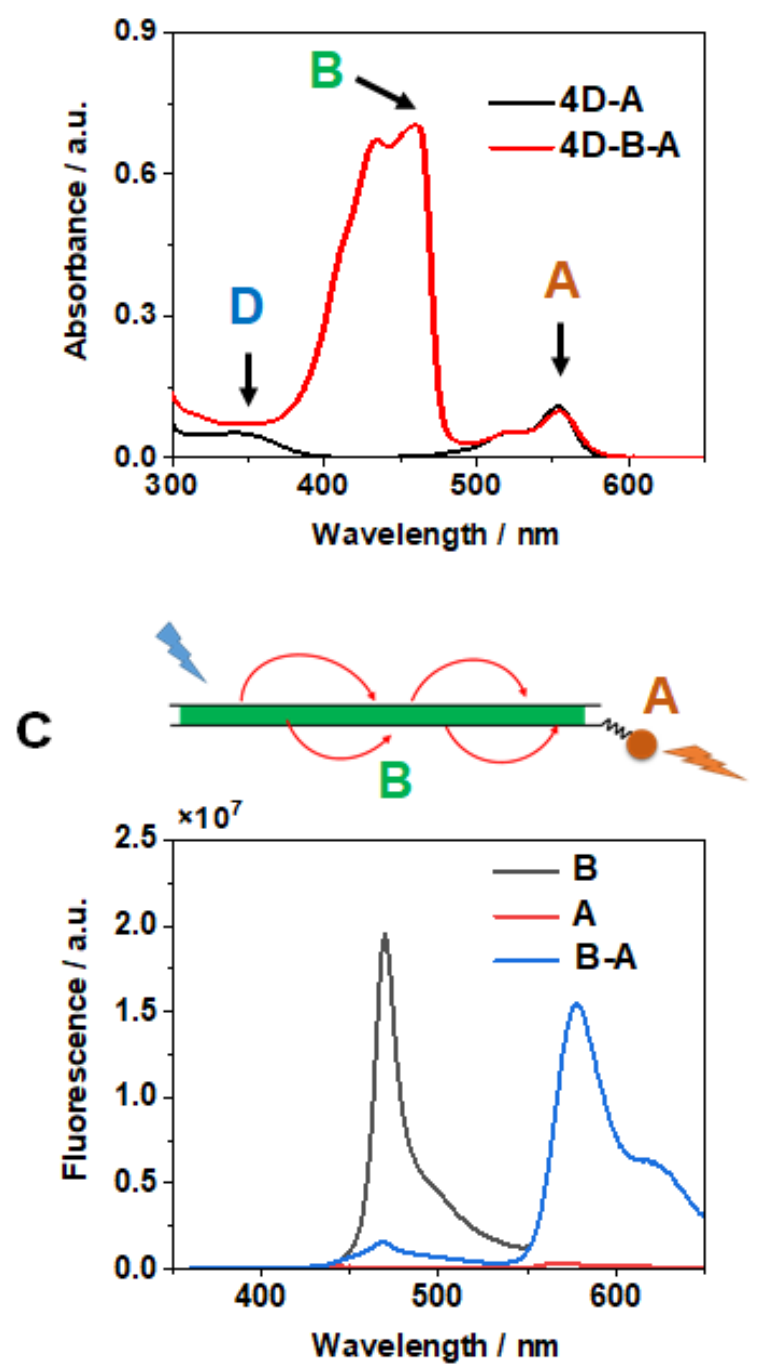
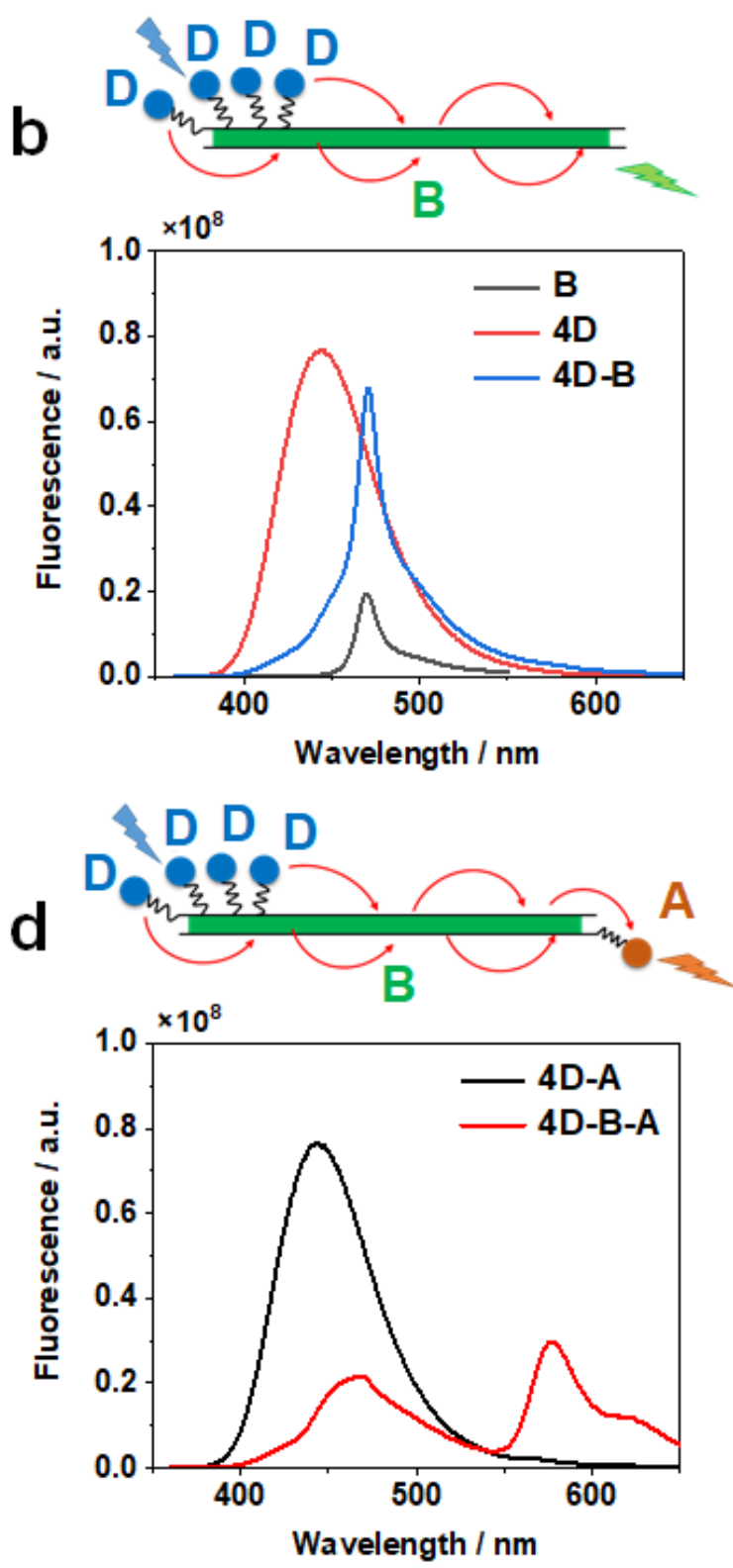

Figure S11. Energy transfer study of 4D-B-A system based on 60 bp random sequence template. a. Absorption spectra of constructs 4D-A (black line) and 4D-B-A (red line). b. Steady-state fluorescence spectra of construct B (black line), 4D (red line) and 4D-B (blue line). c. Steady-state fluorescence spectra of construct B (black line), A (red line) and B-A (blue line). d. Steady-state fluorescence spectra of construct 4D-A (black line) and 4D-B-A (red line). All fluorescence experiments were carried out with excitation at $350 \mathrm{~nm}$. 
a
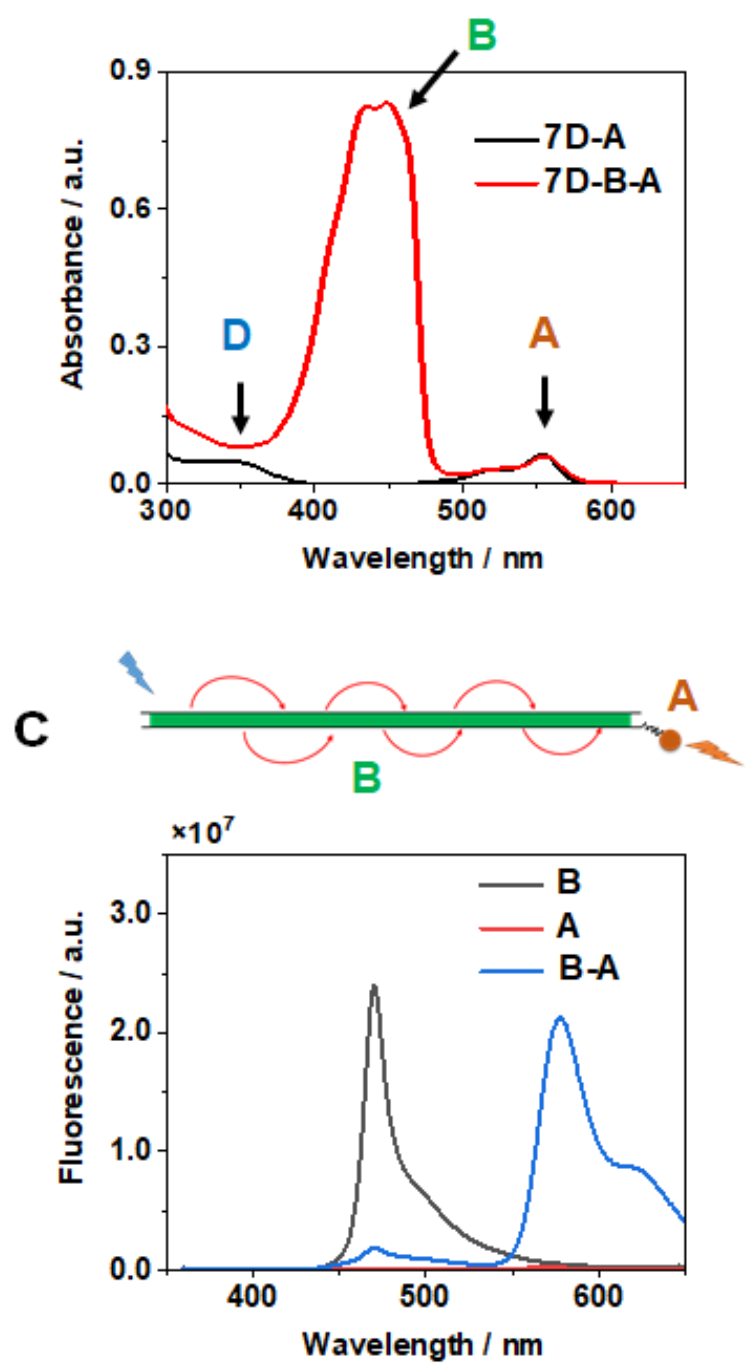

b
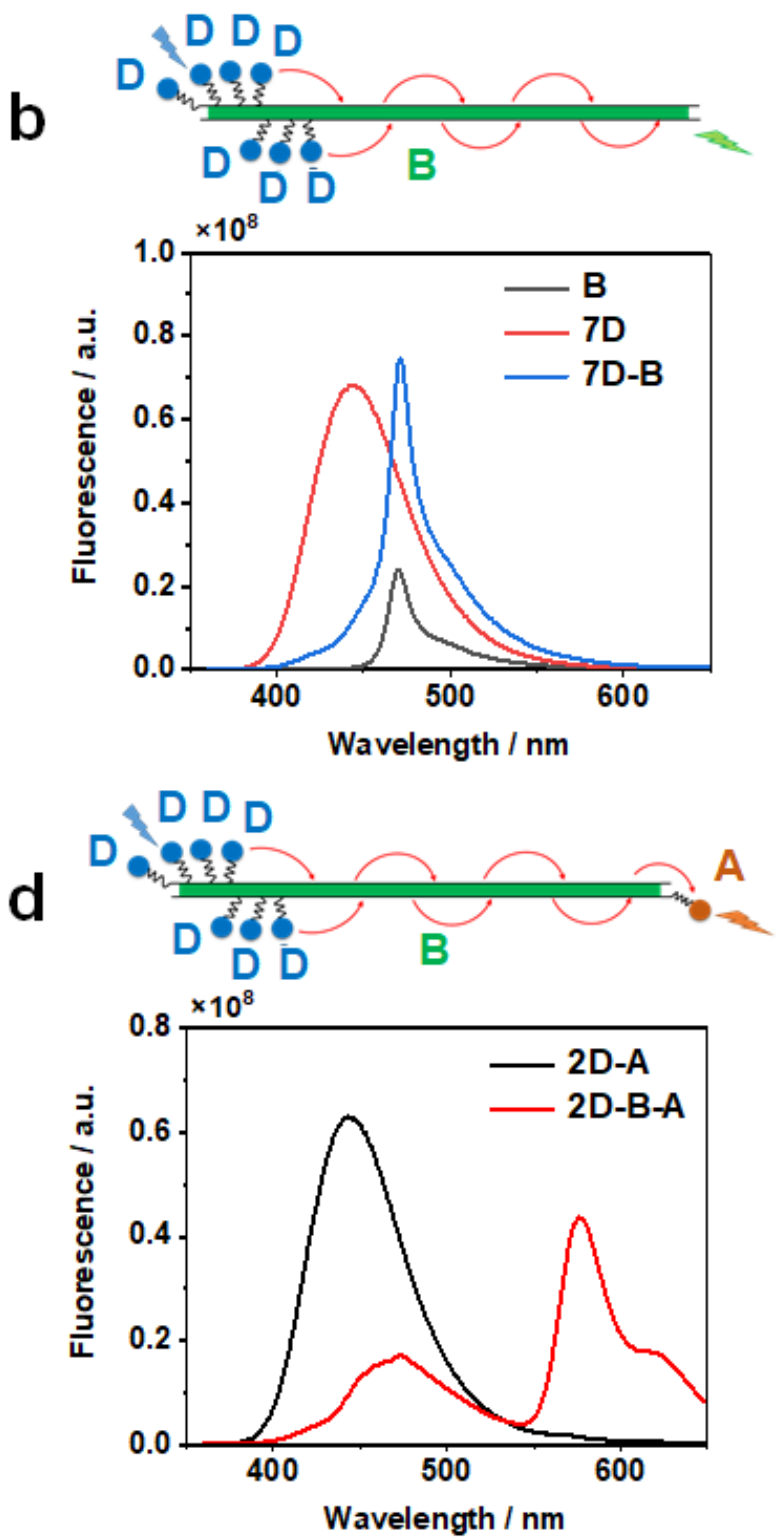

Figure S12. Energy transfer study of 7D-B-A system based on $100 \mathrm{bp}$ random sequence template. a. Absorption spectra of constructs 7D-A (black line) and 7D-B-A (red line). b. Steady-state fluorescence spectra of construct B (black line), 7D (red line) and 7D-B (blue line). c. Steady-state fluorescence spectra of construct B (black line), A (red line) and B-A (blue line). d. Steady-state fluorescence spectra of construct 7D-A (black line) and 7D-B-A (red line). All fluorescence experiments were carried out with excitation at $350 \mathrm{~nm}$. 

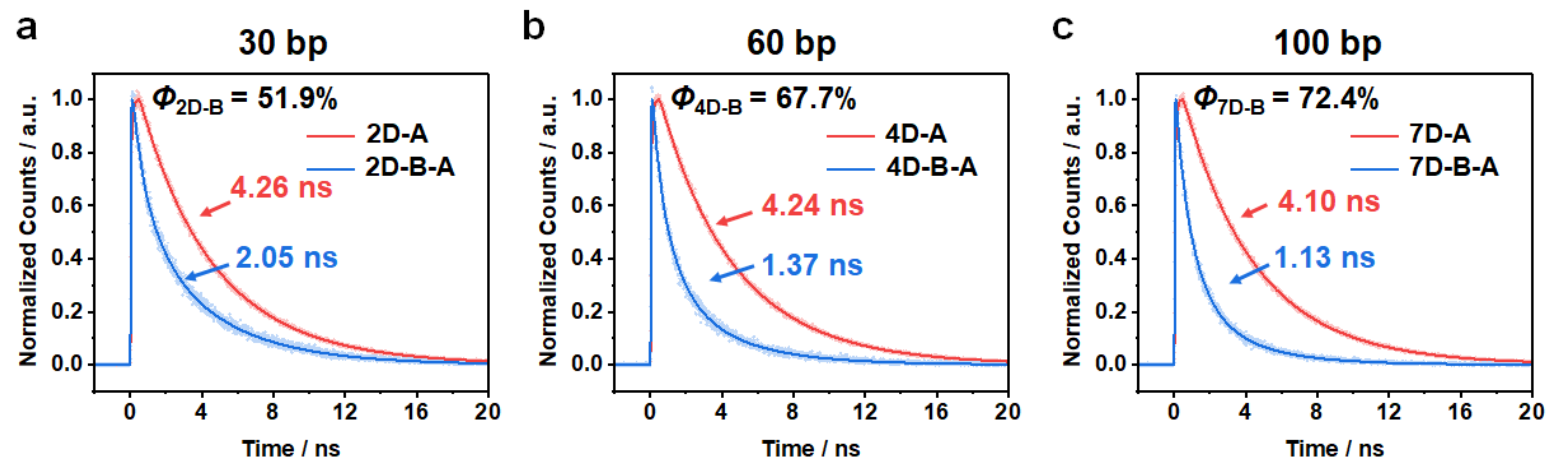

Figure S13. Comparison of D-to-A energy transfer on DNA templates of a. 30 bp, b. 60 bp and c. 100 $\mathrm{bp}$, in all cases with or without the bridge. The fluorescence decay kinetics were monitored at $450 \mathrm{~nm}$ (donor emission wavelength) with excitation at $350 \mathrm{~nm}$. The average fluorescence lifetime $\tau$ of the donor is labeled as red or blue, in the $\mathrm{nD}-\mathrm{A}$ or $\mathrm{nD}-\mathrm{B}-\mathrm{A}$ construct, respectively. The $\mathrm{D}$-to-B energy transfer efficiencies are calculated using $1-\tau(\mathrm{nD}-\mathrm{B}-\mathrm{A}) / \tau(\mathrm{nD}-\mathrm{A})$.
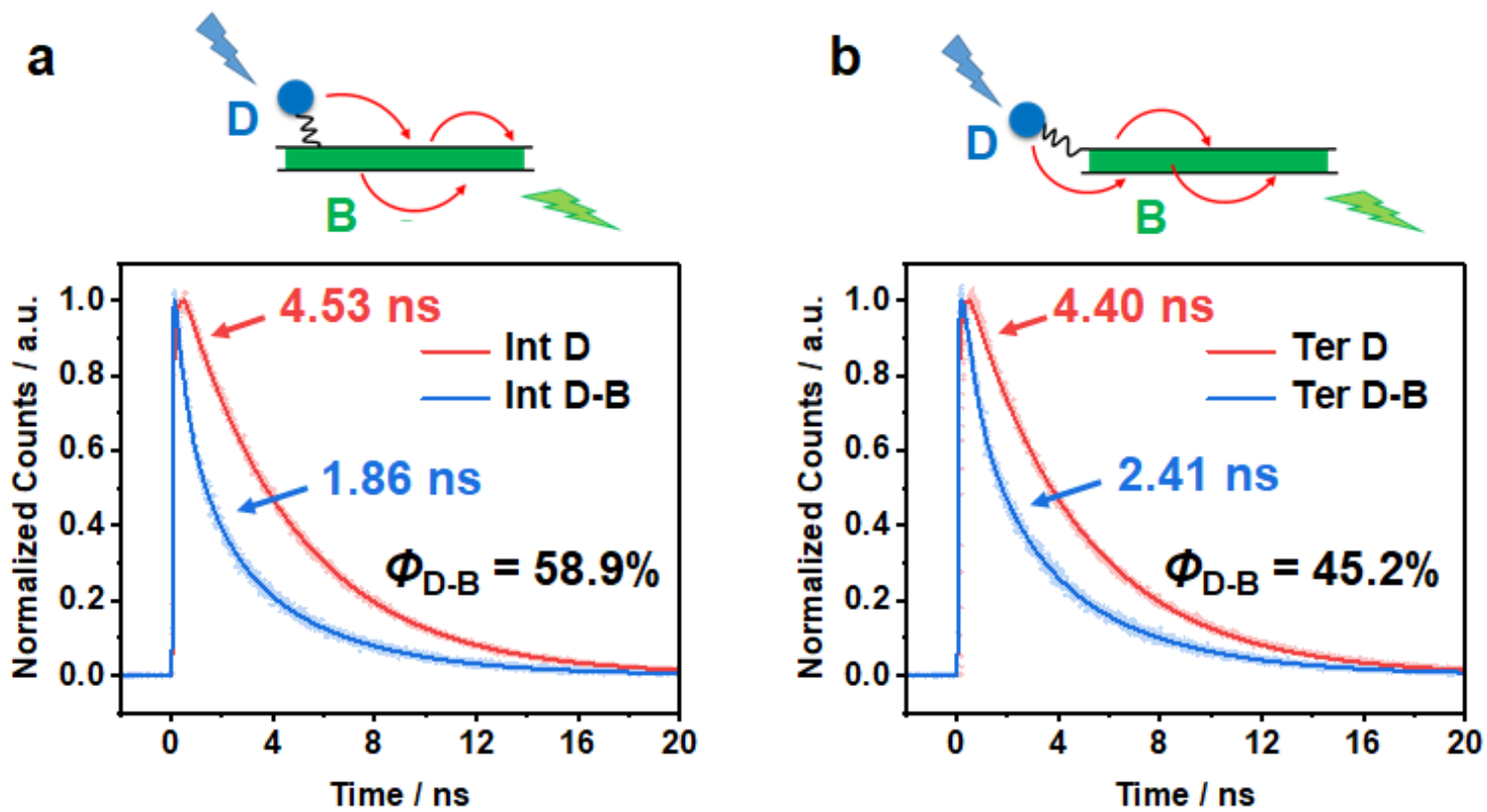

Figure S14. Effect of donor's modification site on the DNA template for the D-to-B energy transfer efficiency. The fluorescence decay kinetics of D (red line) and D-B (blue line) constructs are monitored at $450 \mathrm{~nm}$ (donor emission wavelength) with excitation at $350 \mathrm{~nm}$ for energy transfer constructs with a. internally modified donor molecule (Int D) and b. 5'-end modified donor molecule (Ter D). The average fluorescence lifetime $\tau$ of the donor in the $\mathrm{D}$ and $\mathrm{D}-\mathrm{B}$ construct is labeled as red and blue, respectively. The D-to-B energy transfer efficiencies are calculated using $1-\tau(\mathrm{nD}-\mathrm{B}) / \tau(\mathrm{nD})$. 


\section{Section 3. DNA sequences and characterization of DNA-dye conjugation}

\subsection{DNA sequence information}

Table S1 ssDNA sequences for spectral study of K21 dye aggregates

\begin{tabular}{|c|c|c|}
\hline \multicolumn{2}{|c|}{ DNA template } & Sequence \\
\hline \multirow{2}{*}{$\begin{array}{l}\operatorname{Poly}(\mathrm{A}) 8 / \text { poly }(\mathrm{T}) 8 \\
\text { (14 bp) }\end{array}$} & $\operatorname{Poly}(\mathrm{A})_{8}$ & CGC AAA AAA AAC GC \\
\hline & $\operatorname{Poly}(\mathrm{T})_{8}$ & GCG TTT TTT TTG CG \\
\hline \multirow{2}{*}{$\begin{array}{l}\left.\operatorname{Poly}(\mathrm{A})_{16} / \operatorname{poly}_{(\mathrm{T})}\right)_{16} \\
(22 \mathrm{bp})\end{array}$} & Poly $(\mathrm{A})_{16}$ & CGC AAA AAA AAA AAA AAA ACG C \\
\hline & $\operatorname{Poly}(\mathrm{T})_{16}$ & $\begin{array}{l}\text { GCG TTT TTT TTT TTT TTT TGC G } \\
\end{array}$ \\
\hline \multirow{2}{*}{$\begin{array}{c}\operatorname{Poly}(\mathrm{A})_{24} / \operatorname{poly}(\mathrm{T})_{24} \\
(30 \mathrm{bp})\end{array}$} & $\operatorname{Poly}(\mathrm{A})_{24}$ & CGC AAA AAA AAA AAA AAA AAA AAA AAA CGC \\
\hline & $\operatorname{Poly}(\mathrm{T})_{24}$ & GCG TTT TTT TTT TTT TTT TTT TTT TTT GCG \\
\hline \multirow{2}{*}{$\begin{array}{c}\text { Poly(GC)8 } \\
(22 \text { bp) }\end{array}$} & Poly(GC)8-1 & GCG GCG CGC GCG CGC GCG CGC G \\
\hline & Poly(GC) $)_{8}-2$ & CGC GCG CGC GCG CGC GCG CCG C \\
\hline \multirow{2}{*}{$\begin{array}{l}\text { Random }_{16} \\
(22 \mathrm{bp})\end{array}$} & Random $_{16-1}$ & GCA CTA AGT CGC TGT ATC TGA G \\
\hline & Random $_{16-2}$ & CTC AGA TAC AGC GAC TTA GTG C \\
\hline
\end{tabular}

Table $\mathbf{S 2}$ ssDNA sequences for energy transfer constructs

\begin{tabular}{|c|c|c|}
\hline \multicolumn{2}{|c|}{ Energy transfer constructs } & Sequence $^{*}$ \\
\hline \multirow{2}{*}{ D-B-A } & $\mathrm{NH}_{2}-24 \mathrm{~A}-1 \mathrm{D}$ & $\mathrm{NH}_{2}$-ACG CAA AAA AAA AAA AAA AAA AAA AAA ACG C \\
\hline & $\mathrm{NH}_{2}-24 \mathrm{~T}-1 \mathrm{D}$ & $\mathrm{NH}_{2}$-TGC GTT TTT TTT TTT TTT TTT TTT TTT TGC G \\
\hline \multirow{4}{*}{ 2D-B-A } & $\left(\mathrm{NH}_{2}\right)_{2}-24 \mathrm{~A}-2 \mathrm{D}$ & $\mathrm{NH}_{2}$-ACC TCA AAA AAA AAA AAA AAA AAA AAA AAC GC \\
\hline & $\mathrm{NH}_{2}-24 \mathrm{~T}-2 \mathrm{D}$ & $\mathrm{NH}_{2}$-TGCG TTT TTT TTT TTT TTT TTT TTT TTT GAGGT \\
\hline & $\left(\mathrm{NH}_{2}\right)_{2}-30 \mathrm{R} 1$ & $\mathrm{NH}_{2}$-ACT CCA TCT ATT CTA CGC CGA CTC AAC GCT \\
\hline & Thiol-30R2 & Thiol-AGC GTT GAG TCG GCG TAG AAT AGA TGG AGT \\
\hline \multirow{2}{*}{ 4D-B-A } & $\left(\mathrm{NH}_{2}\right)_{4}-60 \mathrm{R} 1$ & $\begin{array}{l}\text { NH } \mathrm{NH}_{2} \text {-ACT CCA TCT ATT AAT AAA CCG ACT CGT AAC GCT GCG } \\
\text { GCT GGC ATG GTG ACC TAC CTC ACT }\end{array}$ \\
\hline & Thiol-60R2 & $\begin{array}{l}\text { Thiol-AGT GAG GTA GGT CAC CAT GCC AGC CGC AGC GTT } \\
\text { ACG AGT CGG TTT ATT AAT AGA TGG AGT }\end{array}$ \\
\hline \multirow{2}{*}{ 7D-B-A } & $\left(\mathrm{NH}_{2}\right)_{4}-100 \mathrm{R} 1$ & $\begin{array}{l}\mathrm{NH}_{2} \text {-ACT CCA TCT ATT AAT AAA CCG ACT CGC AAC GCT GCG } \\
\text { GCT GGC ATG GTG ACC TAC CTC GCC GTT GAT GTG AAA AGG } \\
\text { GCC GAT GTA CTT TCG TAA ACT CAG A }\end{array}$ \\
\hline & $\left(\mathrm{NH}_{2}\right)_{3}-100 \mathrm{R} 2-$ Thiol & $\begin{array}{l}\text { Thiol-TCT GAG TTT ACG AAA GTA CAT CGG CCC TTT TCA CAT } \\
\text { CAA CGG CGA GGT AGG TCA CCA TGC CAG CCG CAG CGT TGC } \\
\text { GAG TCG GTT TAT TAA TAG ATG GAG T }\end{array}$ \\
\hline Ter D-B & $\mathrm{NH}_{2}-24 \mathrm{~A}-\mathrm{TerD}$ & $\mathrm{NH}_{2}$-ACC TCA AAA AAA AAA AAA AAA AAA AAA AAC GC \\
\hline Int D-B & $\mathrm{NH}_{2}-24 \mathrm{~A}-\mathrm{IntD}$ & ACC TCA AAA AAA AAA AAA AAA AAA AAA AAC GC \\
\hline
\end{tabular}

* Those Ts marked with red are internal amino group modification sites. 


\subsection{Fluorophore-DNA conjugation}
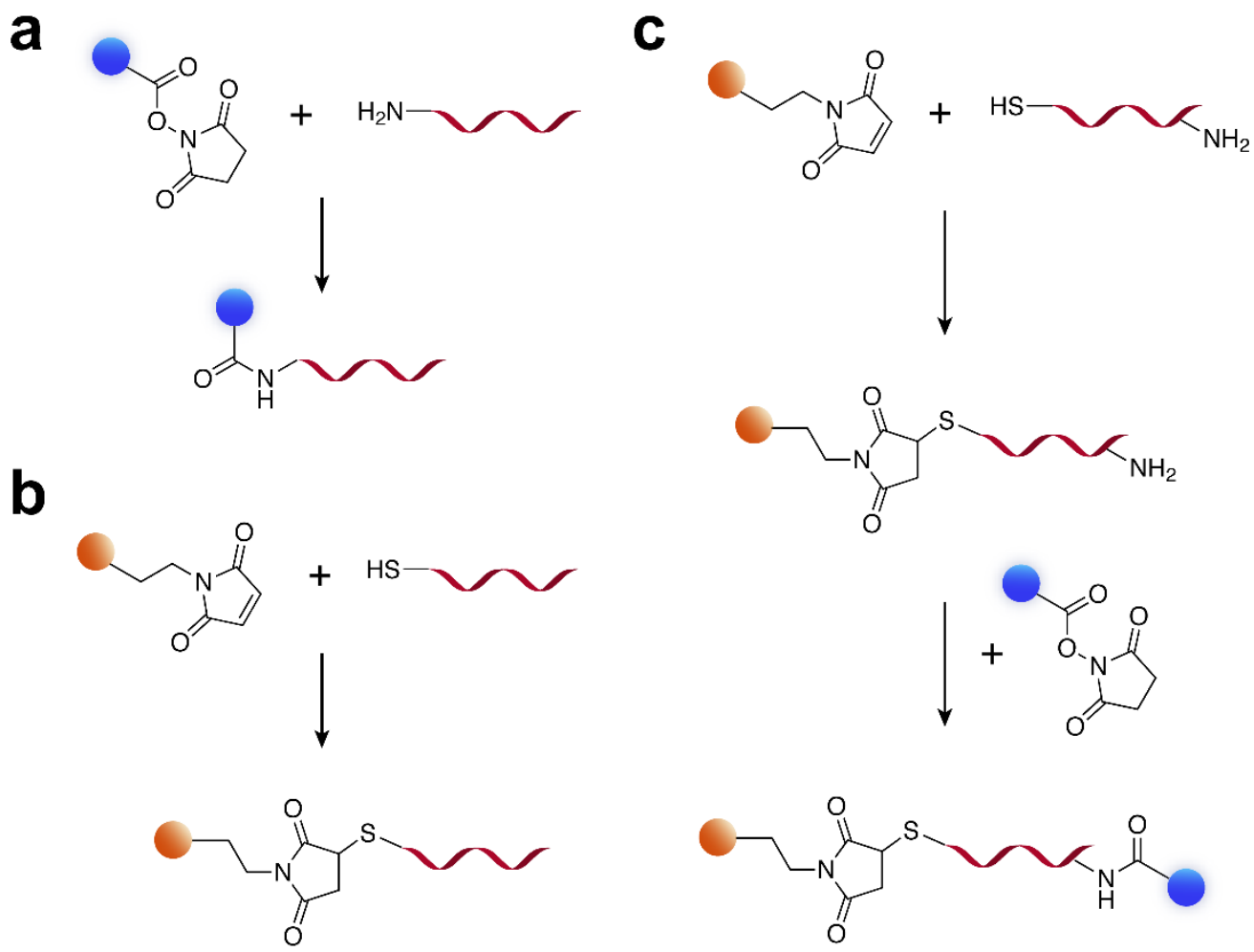

Scheme S1. Schematic diagrams of the fluorophore-DNA conjugation reactions. a. Synthesis of fluorophore-DNA conjugates via amine-NHS ester conjugation. The amine-modified DNA strand was mixed with fluorophore-NHS ester to produce fluorophore labeled DNA. The amino modification can be at the 5'-terminal of the DNA or internally modified on the methyl group of a selected Thymine. $\mathbf{b}$. Synthesis of dye-DNA conjugates via thiol-maleimide conjugation. The thiol group modified DNA reacted with fluorophore-maleimide to form fluorophore-DNA conjugate. c. The dual fluorophores (AF350, blue and AF555 orange) labelled DNA strand was synthesized by using a thiol group and amino group dually modified DNA to react with AF555-maleimide firstly and then react with AF350-NHS ester. 
3.3 MALDI-TOF mass spectra

a

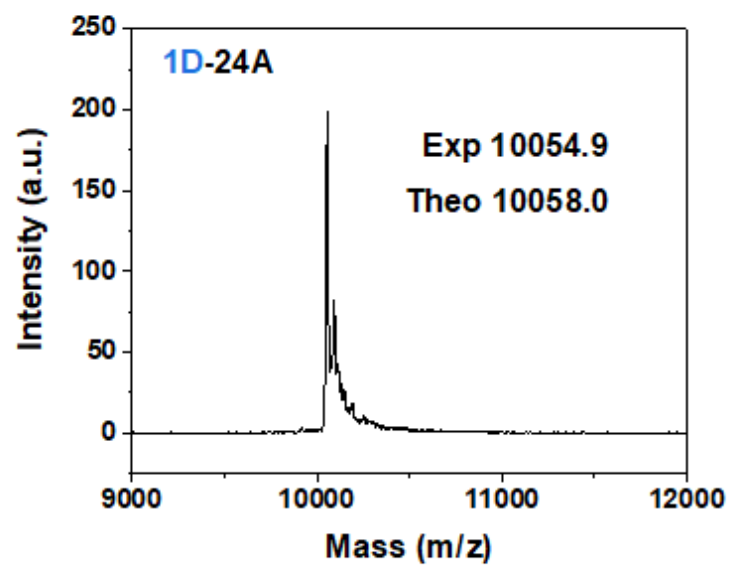

C

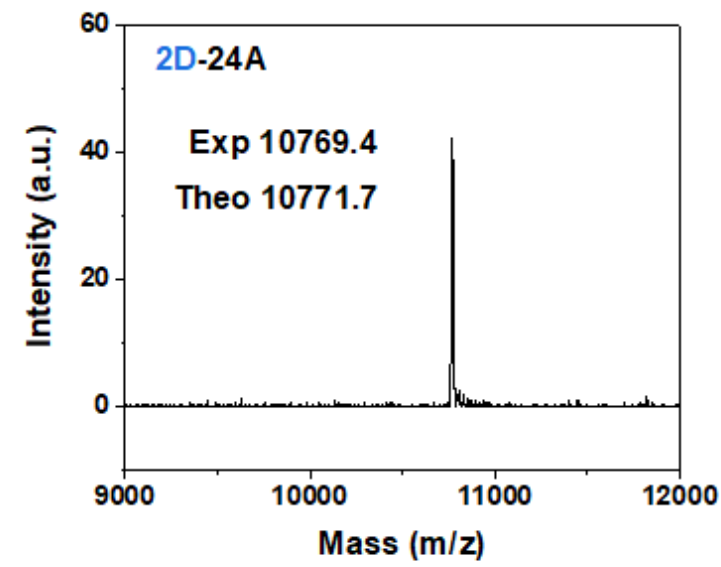

e

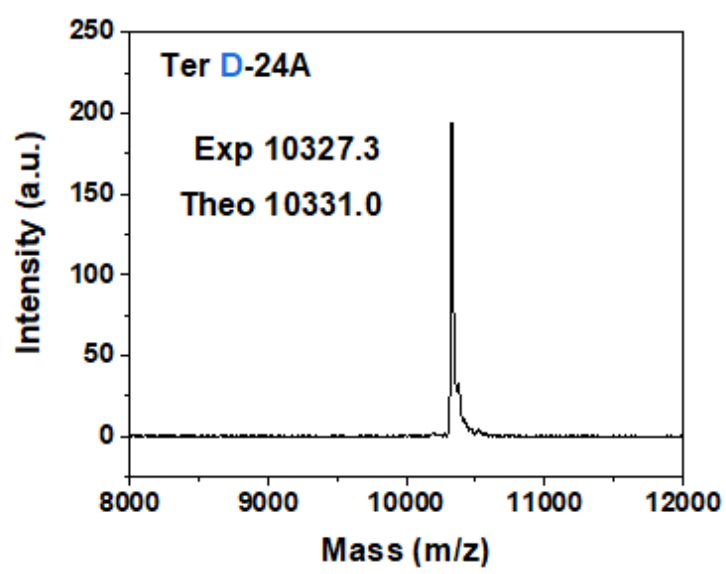

b

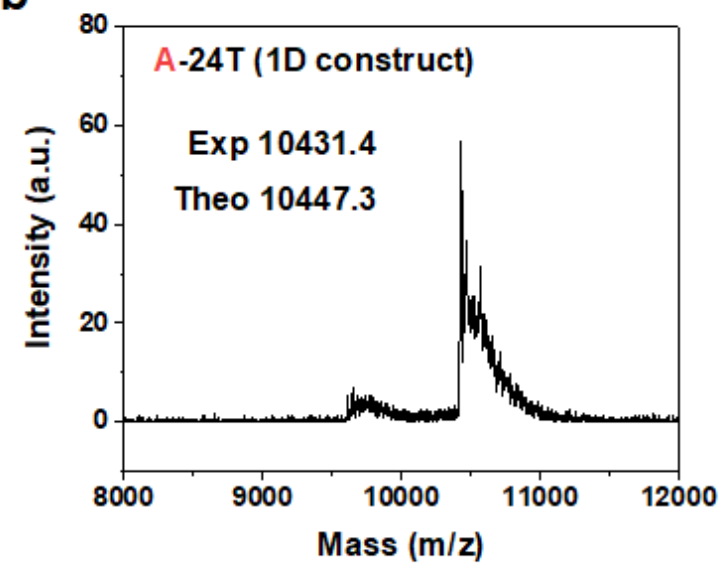

d

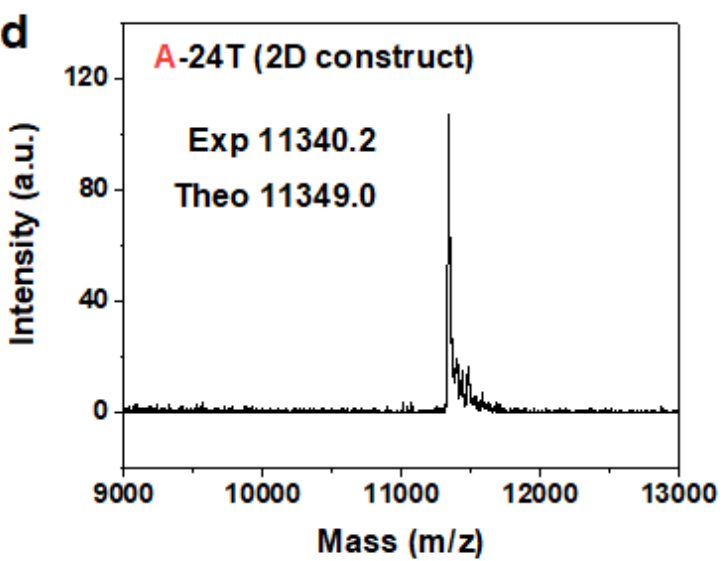

$\mathbf{f}$

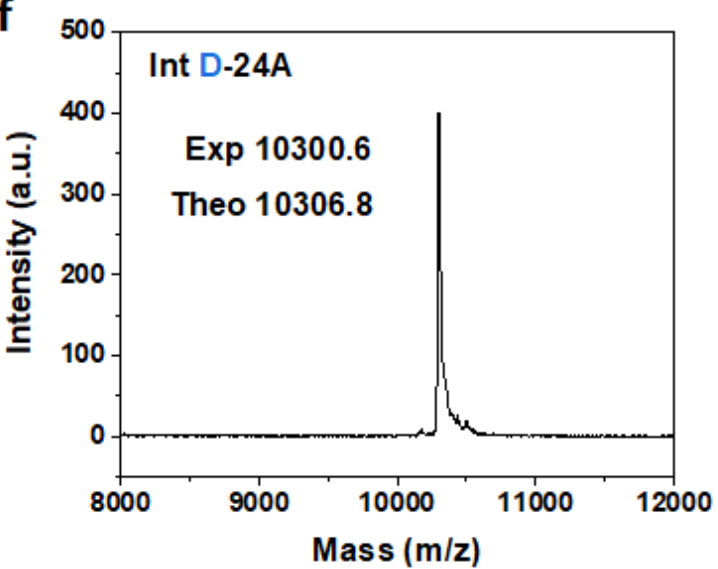

Figure S15. Mass spectrometry analysis of the fluorophore-DNA conjugates on poly $(A)_{24} / \operatorname{poly}(T)_{24}$. a. $1 \mathrm{D}-24 \mathrm{~A}$ and b. A-24T for preparing the $1 \mathrm{D}$ construct. c. $2 \mathrm{D}-24 \mathrm{~A}$ and d. A-24T for preparing the 2D construct. e. D-24A for terminally donor-modified construct. f. D-24A for internally donor-modified construct. The experimental mass (Exp) and theoretical values (Theo) are labelled, confirming the success synthesis of these strands and purity of the sample. 
a

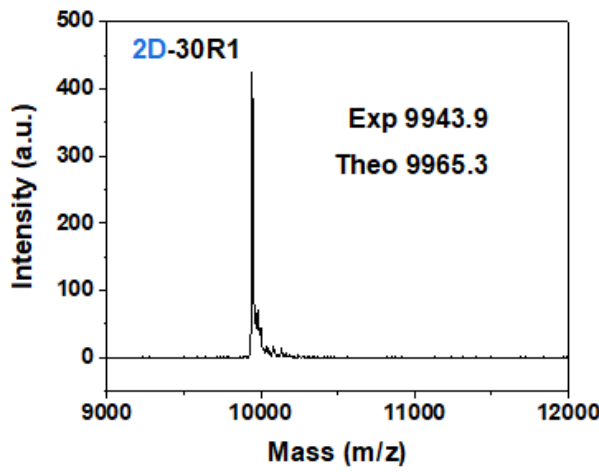

C

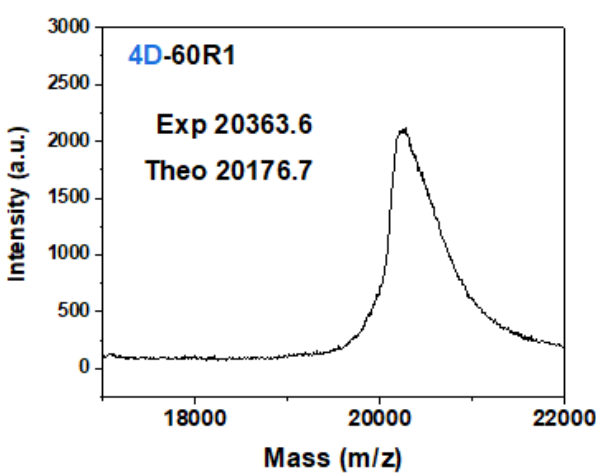

e

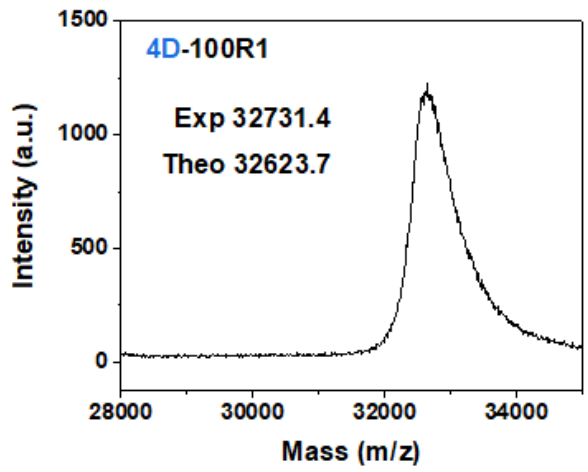

g

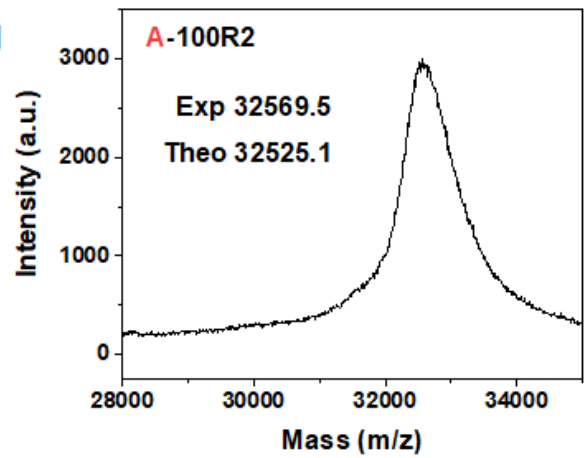

b
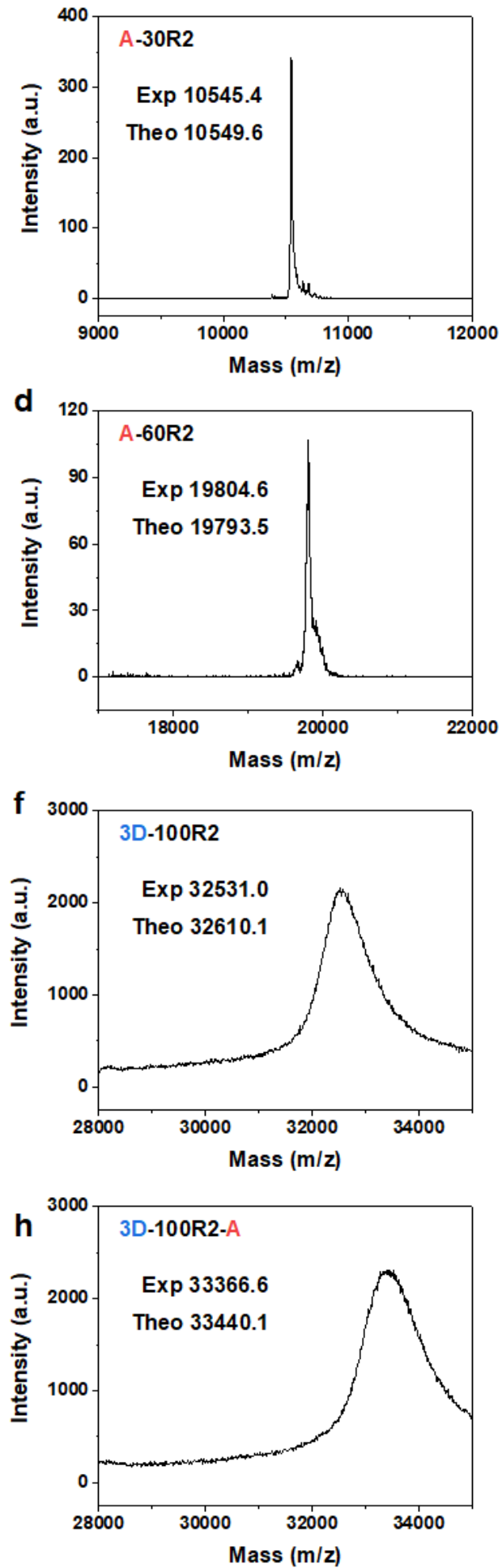

Figure S16. Mass spectrometry analysis of the fluorophore-DNA conjugates on random sequence DNAs. a. 2D-30R1 and b. A-30R2 for 30 bp DNA template. c. 4D-60R1 and d. A-60R2 for 60 bp DNA template. e. 4D-100R1, f. 3D-100R2, f. A-100R2 and g. 3D-100R2-A for $100 \mathrm{bp}$ DNA template. The experimental results (Exp) and the corresponding theoretical values (Theo) are labelled. 


\section{Section 4. Calculation of energy transfer efficiency \\ 4.1 nD-to-B energy transfer efficiency $\phi_{\mathrm{nD}-\mathrm{B}}$}

The nD-to-B energy transfer efficiency $\phi_{\mathrm{nD}-\mathrm{B}}$ was calculated based on the steady-state fluorescence spectroscopy and time-correlated single photon counting (TCSPC) measurements of the $\mathrm{nD}-\mathrm{A}$ and $\mathrm{nD}-\mathrm{B}-$ A constructs. Because the significant spectral overlap of the emission from $\mathrm{D}$ and $\mathrm{B}$, it is hard to obtain clear donor emission from the $\mathrm{nD}-\mathrm{B}$ constructs.

In a typical steady-state fluorescence measurement, the $\mathrm{nD}-\mathrm{A}$ construct $(55 \mu \mathrm{L}, 4 \mu \mathrm{M})$ was excited at $350 \mathrm{~nm}$. A $1.7 \mu \mathrm{L} \mathrm{K} 21$ dye stock solution was added (1.67 mM in DMSO) to the same sample giving the final $\mathrm{K} 21$ dye concentration of $50 \mu \mathrm{M}$ to form the $\mathrm{nD}-\mathrm{B}-\mathrm{A}$ construct. The energy transfer efficiency $\phi_{\mathrm{nD}-\mathrm{B}}$ was calculated according to

$$
\phi_{\mathrm{nD}-\mathrm{B}}=1-\frac{I_{\mathrm{nD}-\mathrm{B}-\mathrm{A}}}{I_{\mathrm{nD}-\mathrm{A}}}
$$

where $I_{\mathrm{nD}-\mathrm{A}}$ and $I_{\mathrm{nD}-\mathrm{B}-\mathrm{A}}$ are the integrated area of donor (D, AF350) fluorescence emission between $380 \mathrm{~nm}$ to $500 \mathrm{~nm}$ in the $\mathrm{nD}-\mathrm{A}$ and $\mathrm{nD}-\mathrm{B}-\mathrm{A}$ constructs, respectively. The fluorescence spectra were corrected for the internal absorption effect using the respective absorption spectra as we described in the Section 1.6a.

Similarly, in a typical TCSPC measurement, nD-A construct $(55 \mu \mathrm{L}, 4 \mu \mathrm{M})$ was excited at $350 \mathrm{~nm}$ and monitored at $450 \mathrm{~nm}$. And then a $1.7 \mu \mathrm{L} \mathrm{K} 21$ dye stock solution (1.67 mM in DMSO) was mixed with the $\mathrm{nD}-\mathrm{A}$ sample to form the $\mathrm{nD}-\mathrm{B}-\mathrm{A}$ construct with a $\mathrm{K} 21$ dye concentration of $50 \mu \mathrm{M}$. The $\mathrm{nD}-\mathrm{B}-$ A construct was also excited and monitored at $350 \mathrm{~nm}$ and $450 \mathrm{~nm}$, respectively. The average fluorescence lifetime of donor (D, AF350) at $450 \mathrm{~nm}$ in the $\mathrm{nD}-\mathrm{A}$ and $\mathrm{nD}-\mathrm{B}-\mathrm{A}$ constructs, $\tau_{\mathrm{nD}-\mathrm{A}}$ and $\tau_{\mathrm{nD}-\mathrm{B}-\mathrm{A}}$, were obtained from fitting the decay curves and the transfer efficiency $\phi_{\mathrm{nD}-\mathrm{B}}$ was calculated according to

$$
\phi_{\mathrm{nD}-\mathrm{B}}=1-\frac{\tau_{\mathrm{nD}-\mathrm{B}-\mathrm{A}}}{\tau_{\mathrm{nD}-\mathrm{A}}}
$$

where $\tau_{\mathrm{nD}-\mathrm{A}}$ and $\tau_{\mathrm{nD}-\mathrm{B}-\mathrm{A}}$ are the average fluorescence lifetime of donor (D, AF350) at $450 \mathrm{~nm}$ in the $\mathrm{nD}-\mathrm{A}$ and $\mathrm{nD}-\mathrm{B}-\mathrm{A}$ constructs respectively. 


\subsection{B-to-A energy transfer efficiency $\phi_{\mathrm{B}-\mathrm{A}}$}

Similarly, the B-to-A energy transfer efficiency $\phi_{\mathrm{B}-\mathrm{A}}$ was calculated based on the steady-state fluorescence and single photon counting measurements of B and B-A constructs. In a typical steady-state fluorescence measurement, B and B-A constructs were prepared via mixing a bare DNA template and an A-labelled DNA template (both $55 \mu \mathrm{L}, 4 \mu \mathrm{M}$ ) with $1.7 \mu \mathrm{L} \mathrm{K21}$ dye stock solution (1.67 mM in DMSO), respectively, to get the final $\mathrm{K} 21$ dye concentration of $50 \mu \mathrm{M}$ in both samples. The fluorescence spectra of B and B-A constructs were measured with excitation of $350 \mathrm{~nm}$. The energy transfer efficiency $\phi_{\mathrm{B}-\mathrm{A}}$ was calculated according to

$$
\phi_{\mathrm{B}-\mathrm{A}}=1-\frac{I_{\mathrm{B}-\mathrm{A}} / A_{\mathrm{B}-\mathrm{A}}}{I_{\mathrm{B}} / A_{\mathrm{B}}}
$$

where $I_{\mathrm{B}}$ and $I_{\mathrm{B}-\mathrm{A}}$ are the integrated area of fluorescence emission from K21 dye aggregates between 445 $\mathrm{nm}$ to $540 \mathrm{~nm}$, corrected by absorbance at $350 \mathrm{~nm}$ for the B and B-A constructs, $A_{\mathrm{B}}$ and $A_{\mathrm{B}-\mathrm{A}}$, respectively.

In a typical TCSPC measurement, B and B-A constructs were prepared following the protocol described above. The two constructs were exited at $350 \mathrm{~nm}$ and monitored at $475 \mathrm{~nm}$, respectively. The energy transfer efficiency $\phi_{\mathrm{B}-\mathrm{A}}$ was calculated according to

$$
\phi_{\mathrm{B}-\mathrm{A}}=1-\frac{\tau_{\mathrm{B}-\mathrm{A}}}{\tau_{\mathrm{B}}}
$$

where $\tau_{\mathrm{B}}$ and $\tau_{\mathrm{B}-\mathrm{A}}$ are the average fluorescence lifetime of K21 dye aggregates (B) at $475 \mathrm{~nm}$ in the B and B-A constructs respectively. 


\section{Section 5. Estimation of overall through bridge donor-to-acceptor energy transfer efficiency}

(a)

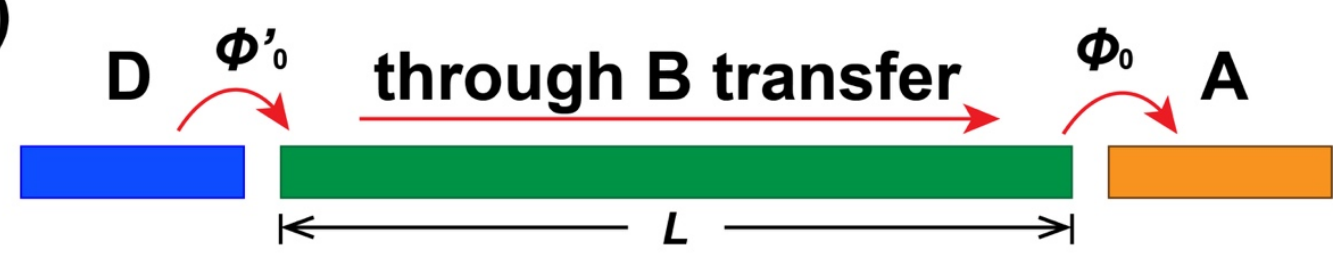

(b)

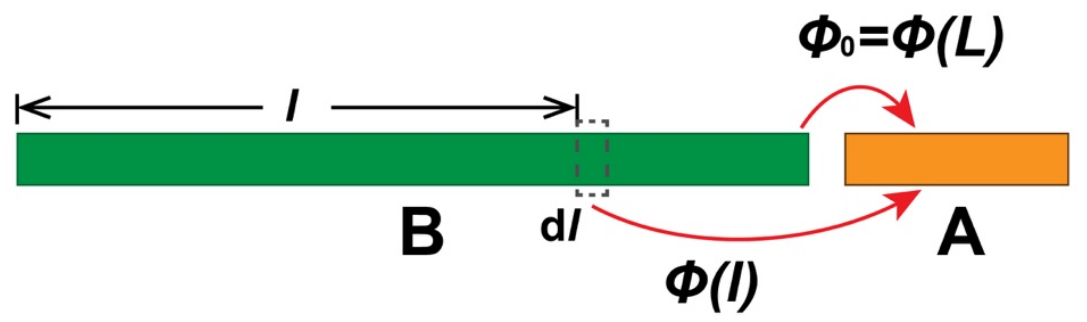

Scheme S2 Energy transfer model for calculation of overall through bridge (B) donor-to-acceptor (D-toA) efficiency. (a) Schematic diagram of three step-wise energy transfer: donor-to-bridge (efficiency of $\phi^{\prime}{ }^{\prime}$ ), through bridge (total length of $L$ ) and end of bridge-to-acceptor (efficiency of $\phi_{0}$ ). (b) Schematic diagram of energy transfer from a certain position $l$ in the aggregate with efficiency of $\phi(l)$.

In the donor-bridge-acceptor (D-B-A) system, the energy transfer process is considered as three steps, including donor-to-bridge, through bridge and bridge-to-acceptor (Scheme S2). To simplify the calculation, the energy transfer from bridge to acceptor only occurs between the most end part of bridge and acceptor with efficient of $\phi_{0}$. Similarly, the donor-to-acceptor energy transfer is considered from the donor to the most beginning part of aggregates with efficiency of $\phi^{\prime}$.

To estimate the energy loss during through bridge transfer, we assume when the energy transfer through the aggregate with total length of $L$, the energy loss ratio over an element distance of $\mathrm{d} l$ is constant. Thus, we expect energy transfer efficiency from an arbitrary position $l$ in the aggregate to the acceptor $\phi(l)$ would be

$$
\phi(l)=\phi_{0} e^{-\frac{L-l}{l_{0}}}
$$

Where $l$ is the distance from donor, $l_{0}$ is the characteristic length where the efficiency dropped by $1 / \mathrm{e}$. The total bridge-to-acceptor energy transfer efficiency $\phi(\mathrm{B}-\mathrm{A})$ that we experimentally measured would be 


$$
\phi(\mathrm{B}-\mathrm{A})=\frac{\int_{0}^{L} \phi_{0} e^{-\frac{L-l}{l_{0}}} d l}{\int_{0}^{L} d l}=\frac{l_{0}}{L}\left(1-e^{-\frac{L}{l_{0}}}\right) \phi_{0}
$$

Using equation (S7), we can obtain $l_{0}$ for constructs with different lengths (30 bp and $100 \mathrm{bp}$ ) of bridge using experimental data and various estimated $\phi_{0}$ (giving in Table S1).

The donor-to-bridge energy transfer would be considered as the direct energy transfer from donor the most beginning part of aggregate $(l \rightarrow 0$ ) and then the excitation energy would migrate over whole aggregates. Thus, the assumed energy transfer efficiency $\phi^{\prime}{ }_{0}$ would be equal with the experimentally measured donor-to-bridge transfer efficiency $\phi(\mathrm{D}-\mathrm{B})$.

Using the assumed model, we can estimate the overall through bridge donor-to-acceptor energy transfer efficiency as

$$
\phi(\mathrm{D}-\mathrm{B}-\mathrm{A})=\phi_{0}^{\prime} \phi(0)=\phi(\mathrm{D}-\mathrm{B}) \phi_{0} e^{-\frac{L}{l_{0}}}
$$

The estimated overall energy transfer efficiencies of 1D, 2D and 100R are given in the Table S1.

Table S1 Summaries of energy transfer efficiencies of different constructs.

\begin{tabular}{cccccccc}
\hline & \multicolumn{2}{c}{$1 \mathrm{D}$} & \multicolumn{3}{c}{ 2D } & \multicolumn{3}{c}{$100 \mathrm{R}$} \\
\cline { 2 - 6 } & Steady-state & TCSPC & Steady-state & TCSPC & & & \\
\hline$\phi(\mathrm{D}-\mathrm{B})$ & $50 \%$ & $45 \%$ & $64 \%$ & $54 \%$ & $72 \%$ & $72 \%$ & $72 \%$ \\
$\phi(\mathrm{B}-\mathrm{A})$ & $96 \%$ & $>96 \%$ & $96 \%$ & $>96 \%$ & $88 \%$ & $88 \%$ & $88 \%$ \\
$\phi_{0}$ & $100 \%$ & $100 \%$ & $100 \%$ & $100 \%$ & $100 \%$ & $95 \%$ & $90 \%$ \\
$L / \mathrm{nm}$ & 9.7 & 9.7 & 9.7 & 9.7 & 32.4 & 32.4 & 32.4 \\
$l_{0} / \mathrm{nm}$ & 120 & 120 & 120 & 120 & 120 & 200 & 650 \\
$\phi(\mathrm{D}-\mathrm{B}-\mathrm{A})$ & $46 \%$ & $42 \%$ & $59 \%$ & $50 \%$ & $55 \%$ & $58 \%$ & $61 \%$ \\
\hline
\end{tabular}




\section{Section 6. A simple kinetics model of D-B-A energy transfer system}

A simple kinetics model is illustrated in Scheme S3. In the model, we studied four species: one species is the 2D-B-A in the ground state, and three species $(\mathrm{X}, \mathrm{Y}, \mathrm{Z})$ in the excited states. $\mathrm{X}, \mathrm{Y}$ and $\mathrm{Z}$ presented the excited donor $\left(\mathrm{D}^{*}\right)$, excited aggregate $\left(\mathrm{B}^{*}\right)$ and excited acceptor $\left(\mathrm{A}^{*}\right)$ respectively. The assumptions are: (1) The energy transfer and excited state decay to ground state processes are all irreversible; (2) the energy transfer processes are considered as the first-order reaction and the energy transfer rate constants for each steps are labeled as $k_{\mathrm{n}}$; (3) the fluorescence decay of each excited species in isolation is considered as single-potential and the fluorescence decay rates of D, B and A are labeled as $\beta_{1}, \beta_{2}$ and $\beta_{3}$ respectively.

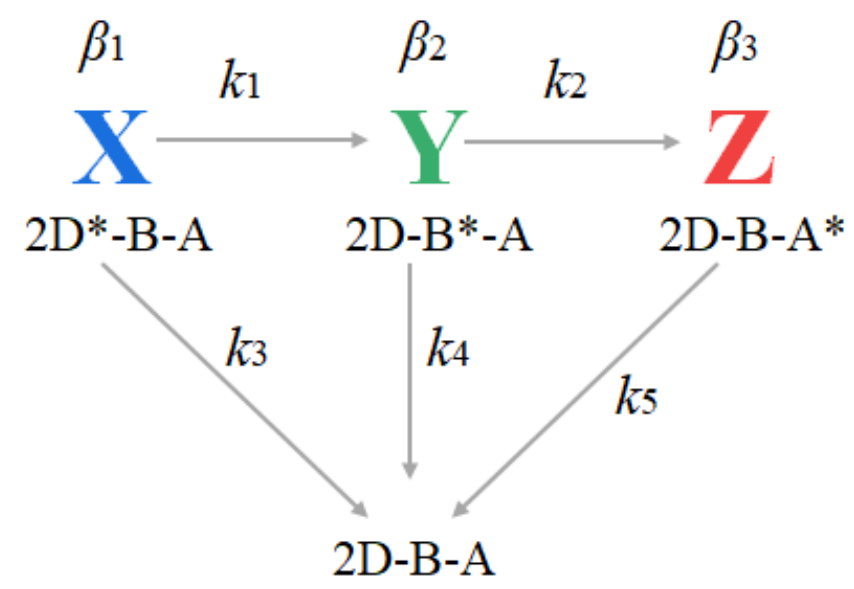

Scheme S3 The kinetics model of energy transfer process in the donor-bridge-acceptor (2D-B-A) system. From the energy transfer Scheme S3, we have:

$$
\begin{gathered}
\frac{d x}{d t}=-\left(k_{1}+k_{3}\right) x \\
\frac{d y}{d t}=k_{1} x-\left(k_{2}+k_{4}\right) y \\
\frac{d z}{d t}=k_{2} y-k_{5} z
\end{gathered}
$$

Based on the fluorescence kinetics fit with exponential decay, we have:

$$
\begin{gathered}
x=x_{0} e^{-\beta_{1} t} \\
y=y_{1} e^{-\beta_{1} t}+y_{2} e^{-\beta_{2} t}
\end{gathered}
$$




$$
z=z_{1} e^{-\beta_{1} t}+z_{2} e^{-\beta_{2} t}+z_{3} e^{-\beta_{3} t}
$$

When $t=0$, we have the initial conditions:

$$
\begin{gathered}
x=x_{0} \quad(\mathrm{~S} 15) \\
y=y_{0}=y_{1}+y_{2} \\
z=z_{0}=z_{1}+z_{2}+z_{3}=0
\end{gathered}
$$

where $x_{0}=3 y_{0}$ because the ratio of absorbance of $\mathrm{D}$ to $\mathrm{B}$ is $3: 1$ in the 2D-B-A construct. From equation (S9)-(S17), we obtained

$$
\begin{gathered}
\beta_{1}=k_{1}+k_{3} \quad(\mathrm{~S} 18) \\
\beta_{2}=k_{2}+k_{4} \quad(\mathrm{~S} 19) \\
\beta_{3}=k_{5} \quad(\mathrm{~S} 20) \\
y_{1}=\frac{k_{1} x_{0}}{\beta_{2}-\beta_{1}} \quad(\mathrm{~S} 21) \\
y_{2}=y_{0}-\frac{k_{1} x_{0}}{\beta_{2}-\beta_{1}} \quad(\mathrm{~S} 22) \quad(\mathrm{S} 23) \\
z_{1}=\frac{k_{2} y_{1}}{\beta_{3}-\beta_{1}} \\
z_{2}=\frac{k_{2} y_{2}}{\beta_{3}-\beta_{2}} \quad(\mathrm{~S} 24) \\
z_{3}=-\frac{k_{2} y_{1}}{\beta_{3}-\frac{\beta_{1}}{\beta_{3}}-\beta_{2}}
\end{gathered}
$$

We determined rate constants from time-resolved fluorescence results of 2D-B-A system showing below.

$$
\begin{aligned}
& \beta_{1}=\frac{1}{\tau_{\mathrm{D}^{*} \mathrm{BA}}}=\frac{1}{1.9} \mathrm{~ns}^{-1}, k_{3}=\frac{1}{\tau_{\mathrm{D}^{*}}}=\frac{1}{4.17} \mathrm{~ns}^{-1}, k_{2}=\frac{1}{\tau_{\mathrm{B} \rightarrow \mathrm{A}}}=\frac{1}{6} \mathrm{ps}^{-1}, k_{4}=\frac{1}{\tau_{\mathrm{B}^{*}}}=\frac{1}{489} \mathrm{ps}^{-1}, \text { and } \\
& k_{5}=\frac{1}{\tau_{\mathrm{A}^{*}}}=\frac{1}{2.44} \mathrm{~ns}^{-1} .
\end{aligned}
$$

Using equation (S18)-(S20), we obtained

$$
k_{1}=\beta_{1}-k_{3}=\frac{1}{3.49} \mathrm{~ns}^{-1}
$$




$$
\begin{gathered}
\beta_{2}=k_{2}+k_{4}=\frac{1}{5.93} \mathrm{ps}^{-1} \\
\beta_{3}=k_{5}=\frac{1}{2.44} \mathrm{~ns}^{-1}
\end{gathered}
$$

Using this kinetics model, we calculated the relative amount of fluorescence emission from donor and acceptor respectively. The fluorescence emission from $\mathrm{D}\left(E m_{\mathrm{D}}\right)$ is

$$
E m_{\mathrm{D}}=\phi_{\mathrm{D}} \frac{k_{3}}{\beta_{1}} x_{0}=0.228 x_{0}
$$

where $\phi_{\mathrm{D}}=0.5$ is the fluorescence quantum yield of donor AF350.

The fluorescence emission from $\mathrm{A}\left(E m_{\mathrm{A}}\right)$ is

$$
E m_{\mathrm{A}}=\phi_{\mathrm{A}} \int_{0}^{\infty} k_{5} z d t=k_{5} \phi_{\mathrm{A}}\left(\frac{z_{1}}{\beta_{1}}+\frac{z_{2}}{\beta_{2}}+\frac{z_{3}}{\beta_{3}}\right)=\frac{k_{2} k_{5}\left(k_{1} x_{0}+\beta_{1} y_{0}\right) \phi_{\mathrm{A}}}{\beta_{1} \beta_{2} \beta_{3}}=0.087 x_{0}
$$

where $\phi_{\mathrm{A}}=0.1$ is the fluorescence quantum yield of acceptor AF555.

Hence, we have

$$
\frac{E m_{\mathrm{D}}}{E m_{\mathrm{A}}}=\frac{0.228 x_{0}}{0.087 x_{0}}=2.62
$$

From the steady-state fluorescence measurements, the ratio of fluorescence emission from $\mathrm{D}$ to $\mathrm{A}$ in $2 \mathrm{D}$ B-A construct is

$$
\frac{I_{\mathrm{D}}}{I_{\mathrm{A}}}=\frac{3.03 \times 10^{8}}{1.46 \times 10^{8}}=2.08
$$

where $I_{\mathrm{D}}$ is the integrated area of donor fluorescence emission in the 2D-B-A construct between $380 \mathrm{~nm}$ to $540 \mathrm{~nm}$, and $I_{\mathrm{A}}$ is the integrated area of acceptor fluorescence emission in the 2D-B-A construct between $560 \mathrm{~nm}$ to $650 \mathrm{~nm}$. From equation (S31) and (S32), two values calculated from steady-state experiment and kinetics model are close, suggesting that we can use such kinetics model to roughly describe the energy transfer process in the 2D-B-A construct and the steady-state results match well with the time-resolved results.

\section{References}

1. Lu, L.; Jones, R. M.; McBranch, D.; Whitten, D., Surface-Enhanced Superquenching of Cyanine Dyes as J-Aggregates on Laponite Clay Nanoparticles. Langmuir 2002, 18 (20), 7706-7713. 